\title{
Anticipating acene-based chromophore spectra with molecular orbital arguments
}

\author{
Timothy J. H. Hele, ${ }^{* \dagger} \dagger$ Eric G. Fuemmeler, $\ddagger$ Samuel N. Sanders, $₫$ Elango \\ Kumarasamy, "Matthew Y. Sfeir, ${ }^{\S}$ Luis M. Campos, ${ }^{*}$, and Nandini Ananth*,‡ \\ †Cavendish Laboratory, JJ Thomson Avenue, Cambridge University, CB3 OHE, UK \\ †Baker Laboratory, 259 East Avenue, Cornell University, Ithaca, NY 14850, USA \\ ФDepartment of Chemistry, Columbia University, New York, NY 10027, USA \\ $\S$ Center for Functional Nanomaterials, Brookhaven National Laboratory, Upton, NY 11973 , \\ $U S A$ \\ E-mail: tjhh2@cam.ac.uk; Icampos@columbia.edu; ananth@cornell.edu
}




\begin{abstract}
Recent synthetic studies on the organic molecules tetracene and pentacene have found certain dimers and oligomers to exhibit an intense absorption in the visible region of the spectrum which is not present in the monomer or many previously-studied dimers. In this article we combine experimental synthesis with electronic structure theory and spectral computation to show that this absorption arises from an otherwise dark charge-transfer excitation 'borrowing intensity' from an intense UV excitation. Further, by characterizing the role of relevant monomer molecular orbitals, we arrive at a design principle that allows us to predict the presence or absence of an additional absorption based on the bonding geometry of the dimer. We find this rule correctly explains the spectra of a wide range of acene derivatives and solves an unexplained structure-spectrum phenomenon first observed seventy years ago. These results pave the way for the design of highly absorbent chromophores with applications ranging from photovoltaics to liquid crystals.
\end{abstract}

\title{
1 Introduction
}

Investigation of the electronic structure and spectra of organic molecules has a long history, dating back to the molecular orbital theories of Hückel ${ }^{1}$ and the oscillator strength sum rules of Thomas, Reiche and Kuhn (TRK). ${ }^{2-4}$ These have been followed by development and application of more sophisticated electronic structure methods ${ }^{5-14}$ and the formulation of structure-spectrum design principles such as Kasha's point-dipole model, ${ }^{15}$ crystallochromy ${ }^{16}$ and the tuning of absorption frequencies (color) by alteration of orbital energy gaps. ${ }^{17}$ Despite this considerable progress, there remains a need for more systematic design rules for the creation of absorbent and tunable chromophores. ${ }^{18-20}$

More recently, there has been a surge of interest in organic chromophores for the development of efficient photovoltaics. ${ }^{21,22}$ In particular, acenes such as tetracene and pentacene possess the unusual and useful ability to undergo singlet fission ${ }^{23-26}$ which, by splitting one 
high-energy exciton into two low-energy triplets, has the potential to substantially increase the efficiency of organic solar cells. ${ }^{27,28}$ This has led to the synthesis and characterization of a large range of dimers and oligomers of tetracene and pentacene, some of which exhibit an intense visible absorption in addition to the monomer $S_{0} \rightarrow S_{1}$ transition, ${ }^{25,29,30}$ whereas others do not. ${ }^{31}$ This additional absorption occurs without any significant change in the intensity or frequency of the lowest-energy monomer $\left(S_{0} \rightarrow S_{1}\right)$ excitation, which is preserved in all these dimers. ${ }^{25,29-31}$ Examining the literature, we find that an interesting dependence of UV-vis spectra on the bonding geometry of acene dimers was first observed in 1948 for dimers of naphthalene (where all the transitions are in the UV), ${ }^{32}$ and has since been observed in many other acene derivatives. ${ }^{33-36}$ Previous computational investigations have assigned this to a $\pi \rightarrow \pi^{*}$ transition $^{33,37}$ but there has been no clear explanation of its presence in some acene derivatives ${ }^{32-36}$ and absence in others. ${ }^{38-45}$

While initially a curiosity, the ability to synthesize acene derivatives that exhibit enhanced visible absorptions may lead to organic photovoltaic materials with increased efficiency. In theory, using a more absorbent chromophore would allow for a decrease in the thickness of a cell while still absorbing the same proportion of solar radiation thereby reducing cost and increasing flexibility. The thinner cell would also allow more diffusing excitons to be extracted as current, increasing efficiency. ${ }^{22,46}$ There is, consequently, a theoretical and practical need to uncover the origin of this new absorption for the design of novel acene-derivative based materials.

In this article we present a joint experimental-theoretical investigation that finds the origin of this new visible absorption is 'intensity borrowing' 47 from an intense UV absorption. The presence or absence of intensity borrowing in dimers can be deduced by examining whether the relevant monomer molecular orbitals have amplitude on the carbon atoms through which the monomers are bonded. We find that the resulting design rule can be used to predict the absorption spectra of a very wide range of acene derivatives, including oligomers with unusual bonding geometries and heteroatom-substituted derivatives. 
We begin by presenting experimental and computed acene spectra in section 2 , finding that Pople-Parr-Pariser (PPP) theory ${ }^{5-8}$ correctly predicts the presence or absence of the extra absorption and its approximate intensity. To explain why this additional absorption is observed in some dimers and not others, in section 3, we use intensity borrowing perturbation theory and PPP theory to analyze the relevant monomer molecular orbitals. In section 4 we apply this analysis to bipentacenes, explaining the origin of the new absorption in some dimers and its absence in others, and we formulate a general design rule for acene derivatives. We show that this design rule correctly predicts the presence or absence of an additional absorption in a large and diverse range of dimers, trimers, and oligomers in section 5 . Conclusions are presented in section 6 .

\section{Acene dimer spectra}

The spectra of acenes is a much-studied area (see, for example, Refs. 8-10,10,12-14,40,48-58) and here we focus on the particular case of covalently-linked dimers and oligomers of acenes. Although we find our results to be widely applicable, we present them in the context of Bis(triisopropylsilylethynyl)pentacene (TIPS-pentacene, Fig. Fig. 1A), a molecule of interest for its extensive applications in photovoltaics. ${ }^{24,25,37,38,59}$

\section{$2.1 \quad$ Experimental spectra}

We start by comparing the experimental spectra of TIPS-pentacene and a recently-reported

dimer, 2,2-bipentacene (22BP), ${ }^{25,59}$ with molecules shown in Fig. 1 and spectra in Fig. 2. The spectra of TIPS-pentacene and 22BP have the familiar features of an acene spectrum: ${ }^{8,12,53} \mathrm{a}$ weak $y$-polarized transition at low frequencies (coordinates defined in Fig. 1A), accompanied by a vibrational stretching progression and a very intense absorption in the near UV (310$350 \mathrm{~nm})$. We note two distinct differences between the spectrum of 22BP and the monomer. First, 22BP exhibits a visible absorption around 500nm with its own vibrational stretching 


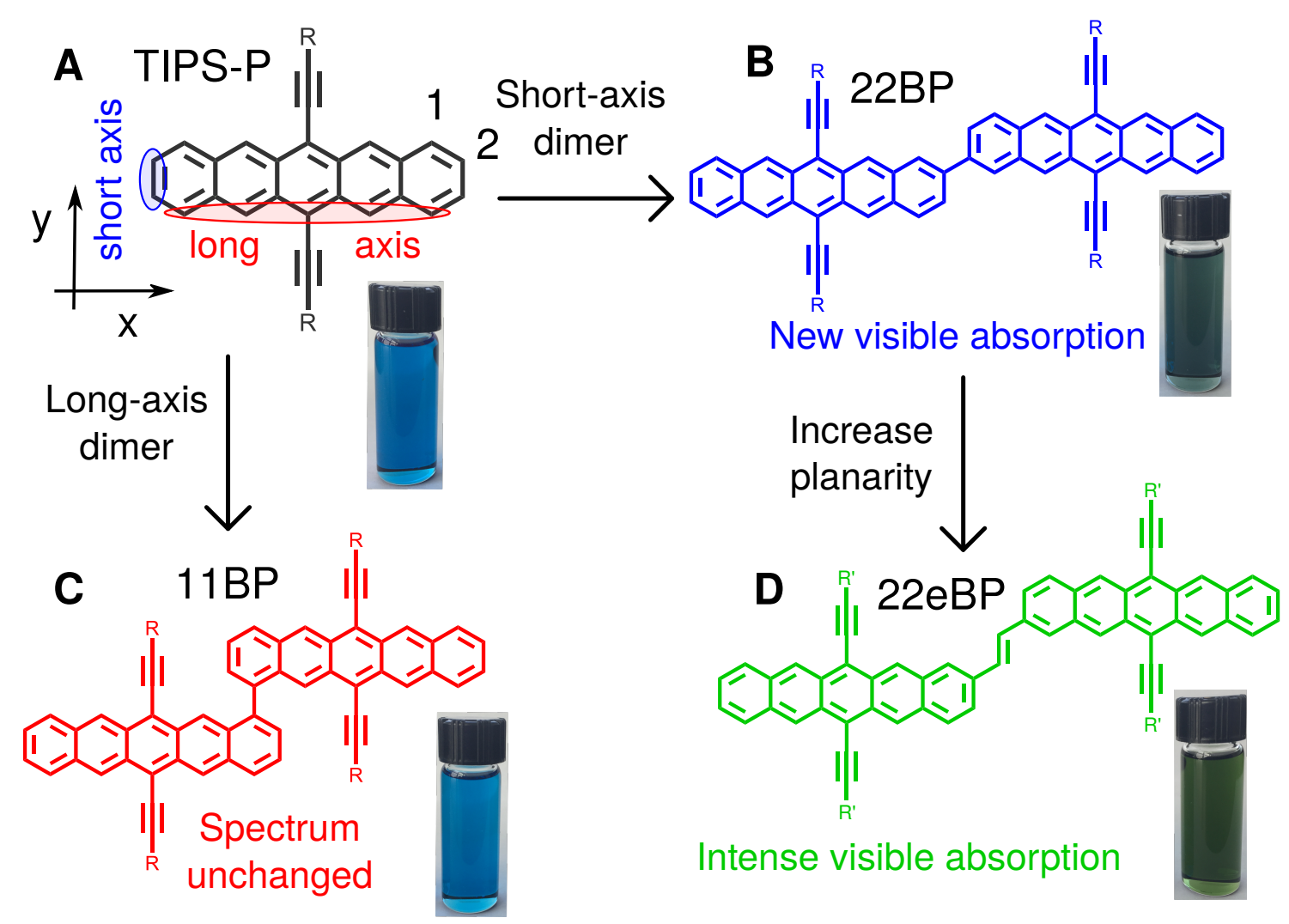

Figure 1: Structures of TIPS-pentacene (A), 2,2'-bipentacene (22BP, B), 1,1'-bipentacene $(11 \mathrm{BP}, \mathbf{C})$ and $2,2^{\prime}$-alkene-linked bipentacene $(22 \mathrm{eBP}, \mathbf{D})$. On TIPS-pentacene the coordinate axes are given along with definitions of short-axis (cata) and long-axis (peri) positions. By each molecule is a picture of an experimental solution in $\mathrm{CHCl}_{3}$, showing how both TIPS-P and $11 \mathrm{BP}$ are light blue, $22 \mathrm{BP}$ is blue-green, and 22eBP is dark green. The corresponding linear absorption spectra are in Fig. 2.

progression. Second, although the lowest energy transition $\left(S_{0} \rightarrow S_{1}\right)$ is unaffected by dimerization, the intensity of the UV absorption decreases and red-shifts. The extinction coefficients in Fig. 2 are plotted per pentacene to show that, in the visible, the $2,2^{\prime}$ dimer is more absorbent than the sum of its parts and not simply an artifact of the chromophore being twice as large.

A previous study focusing on the singlet fission properties of these molecules ${ }^{59}$ considered bonding bulking phenyl groups around the inter-pentacene bond, finding that the size of the new absorption decreased as the twist angle between the two chromophores increased. Similarly, bonding a succession of benzene groups as linkers between the two pentacenes causes the new absorption to decrease in intensity. To investigate the origin of this visible 


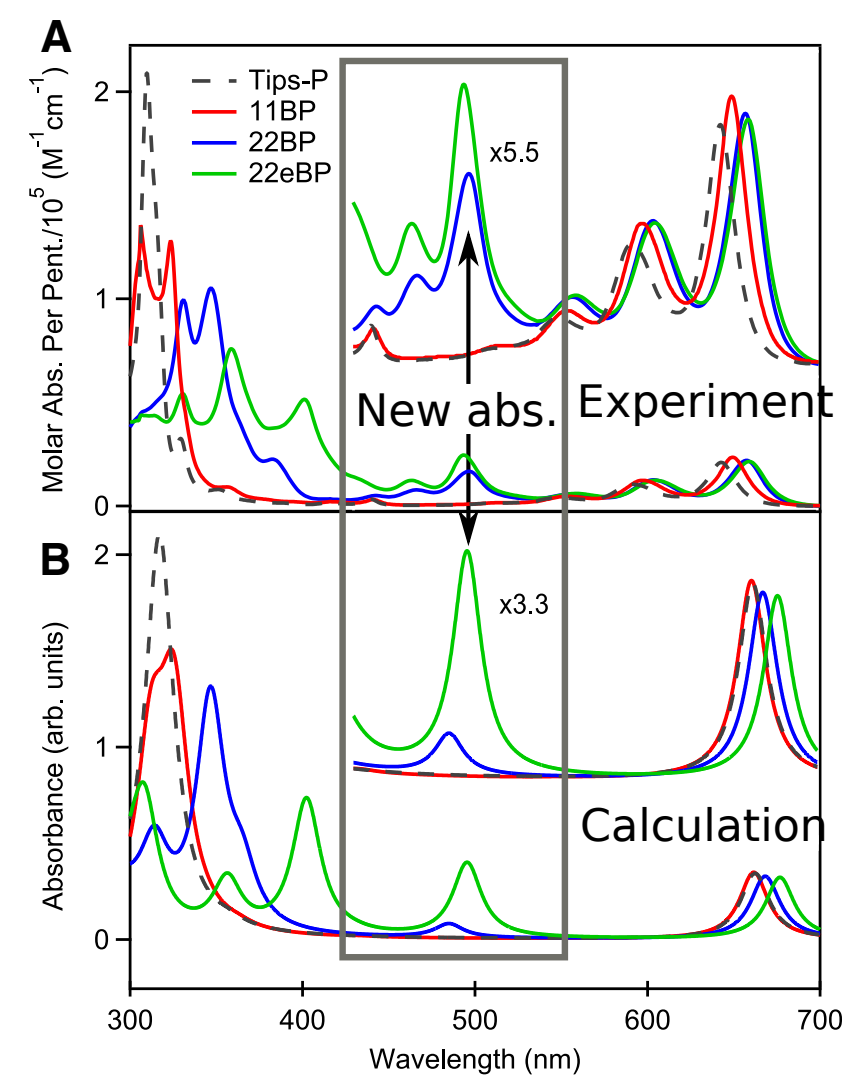

Figure 2: Experimental spectra of TIPS-P (black dashes), 11BP (red), 22BP (blue), and $22 \mathrm{eBP}$ (green) taken in $\mathrm{CHCl}_{3}(\mathbf{A})$ and corresponding computed spectra (using PPP theory) (B), with insets in the visible region. Although the low-energy transition around 650nm is virtually unchanged upon dimerization, the yellow box highlights the emergence of a new absorption around $500 \mathrm{~nm}$, seen in $22 \mathrm{BP}$ and $22 \mathrm{eBP}$ (where it is more intense) but not 11BP. The experimental extinction coefficients and computed spectra are plotted per pentacene to show that $22 \mathrm{BP}$ and $22 \mathrm{eBP}$ are more absorbent in the visible than the sum of their parts. Computed spectra have linebroadening applied but have not been shifted to agree with experiment and do not incude vibrational progressions.

absorption further, we synthesized two more pentacene derivatives (details in the supporting information). We make 1,1'-bipentacene (11BP, Fig. 1C) finding its spectrum in Fig. 2 to be qualitatively identical to the TIPS-P monomer spectrum in the visible, and with a splitting of the high-energy absorption in the UV. We also synthesize a planar analogue of 22BP, 2,2'-ethene-linked bipentacene (22eBP, Fig. 1D) by linking the monomers with an alkene bond, where the alkene bond can be loosely interpreted as a conjugated 'wire' between two chromophores. We find that $22 \mathrm{eBP}$ exhibits the most intense absorption in the visible region of the spectrum among the bipentacenes considered here. 


\subsection{Computed spectra}

Since these are very large chromophores, we employ Pariser-Parr-Pople (PPP) theory ${ }^{5,8,60-62}$ to calculate dimer spectra. PPP theory was developed for the calculation of low-lying excited electronic states of conjugated systems, ${ }^{5,8}$ and its similarity to simple molecular orbital (MO) theories such as Hückel theory allows for a chemically intuitive interpretation of results. ${ }^{60,61}$ Unlike Hückel theory, however, PPP theory includes two-electron interactions (within the neglect of differential overlap (NDO) approximation) such that it correctly accounts for Coulomb and exchange interactions which are required to give accurate excited-state energies. ${ }^{7}$ Computational details are given in the SI where we also benchmark our monomer calculations against high-level multiconfiguational calculations, confirming the suitability of PPP theory.

Examining Fig. 2, we see that the PPP spectra are in good agreement with experimental spectra (the computed energies are not shifted to agree with experiment) and correctly predicts a visible absorption in $22 \mathrm{BP}$ and $22 \mathrm{eBP}$ that is not present in either $11 \mathrm{BP}$ or the monomer. While the visible absorption in 22BP is somewhat weaker and slightly blue-shifted compared to experiment, in 22eBP it is accurately captured, both in terms of absorption frequency and relative intensity. We also see that PPP correctly predicts a Davydov splitting of the 11BP absorption (seen as a peak with a shoulder around 320nm), and a diminution in the height of the UV peaks of $22 \mathrm{BP}$ and $22 \mathrm{eBP}$, although their relative intensities are inaccurate.

From our PPP calculations (verified by high-level calculations reported in the SI), we attribute the dimer excitation around 650nm to a predominantly HOMO $\rightarrow$ LUMO transition. The intense UV peak arises from an in-phase combination of HOMO-3 to LUMO and HOMO to LUMO+3, whose corresponding out-of-phase combination is dark in PPP theory and is seen as a weak absorption at $440 \mathrm{~nm}$ in experiment. Excitations from the HOMO-1 to LUMO and HOMO to LUMO+1 are dipole forbidden, and the excitations from the HOMO-2 to the LUMO and the HOMO to the LUMO+2 are predicted by PPP theory to be $y$-polarized, 
giving a dark out-of-phase state and a bright in-phase state at 358nm and 366nm respectively. The bright state probably corresponds to a shoulder in the intense UV absorption seen experimentally around 350nm.

\section{Elucidating dimer spectra}

We investigate the origin of the visible absorption in 22BP and 22eBP using intensity borrowing perturbation theory, ${ }^{47}$ where we contruct a zeroth-order set of states (corresponding to noninteracting monomers), and examine the perturbation (if any) introduced by dimerization by bonding at different positions.

We begin by noting that textbook arguments ${ }^{63}$ fail to explain the variation in our dimer spectra. Conventional symmetry-breaking arguments fail to explain the extra absorption since both $11 \mathrm{BP}$ and $22 \mathrm{BP}$ are $C_{2}$ and the more symmetric $C_{2 h} 22 \mathrm{eBP}$ has the largest absorption (see Fig. 2). Similarly, a particle-in-a-box model ${ }^{63}$ predicts an intense HOMO to LUMO $S_{1}$ transition, which is inaccurate even for the monomer (Fig. 2), and it also predicts that the lowest-energy transition redshifts and grows in intensity upon enlarging the molecule, which is not observed. Furthermore, solvatochromism cannot explain the extra absorption, since alternant hydrocarbons ${ }^{64}$ such as acenes have no ground-state dipole or multipole. Planarity arguments could also be posited, since the overlap of the $\pi$ systems of the two monomers would be expected to increase with planarity, and 11BP is more twisted (nearly perpendicular) than 22BP $\left(37^{\circ}\right.$ dihedral angle $\left.{ }^{59}\right)$ and planar 22eBP. However, by linking pentacenes via an alkyne linker, a planar 6,6'-bipentacene has been synthesized ${ }^{38}$ and shows no extra absorption in the visible (SI Fig. 1). Consequently, planarity is advantageous but not sufficient.

More sophisticated theories of chromophore interaction include Kasha's point-dipole model, ${ }^{15}$ which can be used to explain the spectra of some acene derivatives. ${ }^{65,66}$ Kasha's model requires two bright excitations to interact, and can therefore explain the splitting of 
the high-energy absorption seen in the UV spectrum of 11BP, but not the emergence of a new absorption in a region of the spectrum where there was previously none. The new dimer absorption cannot result from the monomer $S_{0} \rightarrow S_{1}$ transition around 650nm splitting, since that would result in two absorption peaks with combined intensity equal to that of the monomer, whereas the dimer spectra of $22 \mathrm{BP}$ and $22 \mathrm{eBP}$ are more absorbent in the visible than the sum of their parts (see Fig. 2).

However, intensity borrowing perturbation theory ${ }^{47}$ is promising here, as it describes how dark transitions can 'borrow intensity' from bright states if they are coupled through a perturbation. Since oscillator strength is conserved by the TRK sum rule, ${ }^{2-4,63}$ the resulting new absorption in the spectrum is accompanied by a bright transition losing intensity. This is in keeping with our previous observation on the spectra of $22 \mathrm{BP} / 22 \mathrm{eBP}$ where the appearance of a new visible absorption at 500nm is accompanied by a decrease in intensity of the UV peak. We also find that a previous computational study on $22 \mathrm{BP}$ which investigated its singlet fission properties and not the linear absorption spectrum, suggested the existence of a charge-transfer state around 440nm. ${ }^{59}$ A reasonable hypothesis, is then, that a transition in the visible, possibly this CT excitation, is 'borrowing intensity' from the UV in certain dimers like 22BP and 22eBP but not in 11BP.

\subsection{Intensity borrowing perturbation theory and PPP}

We develop a theory to describe the interactions between the monomer UV excitations and the low-energy CT excitation in a dimer by combining intensity borrowing perturbation theory ${ }^{47}$ and PPP theory. We also characterize the role that dimer bonding geometry plays in modulating the strength of this interaction and, therefore, the appearance (or not) of an additional peak in the absorption spectrum.

We first formally define our system in the language of perturbation theory. Similar to

Kasha ${ }^{15}$ and the ideas of symmetry-adapted perturbation theory, ${ }^{67}$ for two monomers $n$ and 
$m$, the Hamiltonian is

$$
\hat{H}=\hat{H}_{n}+\hat{H}_{m}+\hat{V}_{n m}
$$

where $\hat{H}_{n}$ and $\hat{H}_{m}$ are the Hamiltonian operators of the monomers at infinite separation and $\hat{V}_{n m}=: \hat{V}$ is the intermonomer perturbation. Kasha approximated $\hat{V}$ as a dipole-dipole interaction and assumed all excitations were intra-monomer, ${ }^{15}$ but here we make no a priori assumptions about the nature of the excitations nor the functional form of $\hat{V}$. Instead, we choose to describe the overall system using PPP theory ${ }^{5-8}$ which we have already shown provides an accurate description of the electronic structure of these molecules.

To define the monomer Hamiltonians within PPP theory, ${ }^{5-8,61,68}$ we let $\mathcal{M}$ be the set of atoms on monomer $m$ such that

$$
\begin{aligned}
\hat{H}_{m}= & \sum_{\mu \in \mathcal{M}} \epsilon_{\mu} \hat{n}_{\mu}+U_{\mu \mu} \hat{n}_{\mu, \uparrow} \hat{n}_{\mu, \downarrow}-\sum_{\langle\mu<\nu \in \mathcal{M}\rangle} \sum_{\sigma} t_{\mu \nu}\left(\hat{a}_{\mu \sigma}^{\dagger} \hat{a}_{\nu \sigma}+\hat{a}_{\nu \sigma}^{\dagger} \hat{a}_{\mu \sigma}\right) \\
& +\sum_{\mu<\nu \in \mathcal{M}} \gamma_{\mu \nu}\left(\hat{n}_{\mu}-Z_{\mu}\right)\left(\hat{n}_{\nu}-Z_{\nu}\right)
\end{aligned}
$$

and likewise for monomer $n$, where $\hat{n}_{\mu}$ is the number operator for electrons on atom $\mu$,

$$
\hat{n}_{\mu}=\sum_{\sigma=\{\uparrow, \downarrow\}} \hat{n}_{\mu, \sigma},
$$

where $\hat{n}_{\mu, \sigma}=\hat{a}_{\mu, \sigma}^{\dagger} \hat{\sigma}_{\mu, \sigma}$, and $\hat{a}_{\mu, \sigma}^{\dagger}, \hat{a}_{\mu, \sigma}$ are the creation and annihilation operators respectively for an electron of spin $\sigma$ on atom $\mu$. $Z_{\mu}$ is the local chemical potential, which is 1 for a carbon atom. $\epsilon_{\mu}$ is the on-site energy, which for a purely hydrocarbon chromophore we can set to zero without affecting the energies of excited states. $t_{\mu \nu}$ is the hopping term, where \langle\rangle indicates the summation is over nearest neighbor pairs. $U_{\mu \mu}$ is the on-site (Hubbard) repulsion, and $\gamma_{\mu \nu}$ is the parameterized repulsion between an electron on atom $\mu$ and an electron on atom $\nu$ (for details see SI).

Using the definition of the PPP Hamiltonian $\hat{H}$ for an arbitrary chromophore ${ }^{5-8,61,68}$ and 
Eq. (2), we define the intermonomer perturbation as

$$
\begin{aligned}
\hat{V} & =: \hat{H}-\hat{H}_{n}-\hat{H}_{m} \\
& =-\sum_{\langle\mu \in \mathcal{M}, \nu \in \mathcal{N}\rangle} \sum_{\sigma} t_{\mu \nu}\left(\hat{a}_{\mu \sigma}^{\dagger} \hat{a}_{\nu \sigma}+\hat{a}_{\nu \sigma}^{\dagger} \hat{a}_{\mu \sigma}\right)+\sum_{\mu \in \mathcal{M}, \nu \in \mathcal{N}} \gamma_{\mu \nu}\left(\hat{n}_{\mu}-Z_{\mu}\right)\left(\hat{n}_{\nu}-Z_{\nu}\right)
\end{aligned}
$$

Because the two chromophores are noninteracting at infinite separation, we can solve meanfield variants (Fock operators) of $\hat{H}_{n}$ and $\hat{H}_{m}$ separately for their corresponding molecular orbitals. The monomers are identical, allowing us to choose degenerate orbitals that are localized entirely on monomer $n$ or monomer $m$. These molecular orbitals are denoted $\phi_{n i}$ or $\phi_{m i}$ where the subscript indicates the $i^{\text {th }} \mathrm{MO}$ of monomer $n$ or $m$. In accordance with convention $^{8}$ bonding orbitals (those occupied in the ground state) are numbered $1,2,3, \ldots$ from the HOMO downwards and antibonding orbitals $1^{\prime}, 2^{\prime}, 3^{\prime}, \ldots$ from the LUMO upwards. As noted by Pariser, ${ }^{8}$ care must be taken to be consistent with the relative signs of orbitals in order for the Coulson-Rushbrooke theorem ${ }^{64}$ to be easily applied. For the perturbation analysis of the dimers considered here we choose every monomer orbital to have the same sign on the atom through which it is joined to the other monomer.

We are interested in linear absorption spectra and the dipole moment is a one-electron operator, so we consider only singly excited states in keeping with the original formulation of PPP. We denote single excitations $\left|\Phi_{p i}^{q j^{\prime}}\right\rangle$ where $p$ and $q$ are either $n$ or $m$. We only consider singlet spin-adapted configurations ${ }^{69}$ as triplet excitations are dark for hydrocarbons with minimal spin-orbit coupling. There are two types of single excitations. First, when $p=q,\left|\Phi_{n i}^{n j^{\prime}}\right\rangle$ or $\left|\Phi_{m i}^{m j^{\prime}}\right\rangle$, are intramolecular, local (Frenkel) excitations ${ }^{27,57,59,70}$ that we denote $\operatorname{as}^{70}\left|\operatorname{LE}_{n i}^{n j^{\prime}}\right\rangle=:\left|\Phi_{n i}^{n j^{\prime}}\right\rangle$. For alternant hydrocarbons ${ }^{5,8,64}$ such as the molecules considered in this article, $\left|\mathrm{LE}_{n i}^{n j^{\prime}}\right\rangle$ and $\left|\mathrm{LE}_{n j}^{n i^{\prime}}\right\rangle$ are degenerate and we therefore define 'plus' and 'minus' excitations $^{8}$

$$
\left|\mathrm{LE}_{n i n j}^{ \pm}\right\rangle=\frac{1}{\sqrt{2}}\left(\left|\mathrm{LE}_{n i}^{n j^{\prime}}\right\rangle \pm\left|\mathrm{LE}_{n j}^{n i^{\prime}}\right\rangle\right)
$$


where only 'plus' excitations have nonzero transition dipole moment from the ground state. ${ }^{8}$

The second type of excitation (when $p \neq q$ ), $\left|\Phi_{n i}^{m j^{\prime}}\right\rangle$ or $\left|\Phi_{m i}^{n j^{\prime}}\right\rangle$, are intermolecular, chargetransfer $(\mathrm{CT})$ excitations ${ }^{27,57,70}$ which we denote $\left|\mathrm{CT}_{n i}^{m j^{\prime}}\right\rangle=:\left|\Phi_{n i}^{m j^{\prime}}\right\rangle$. We appreciate that there are varying definitions and nomenclature for CT excitations in the literature, ${ }^{27,70-73}$ and here the definition corresponds to removing an electron from an orbital localized entirely on one monomer and placing it in an orbital localized on another monomer. Using the definition of the dipole moment in PPP theory ${ }^{8}$ and that orbitals on different monomers are spatially disjoint, we note that the CT excitations defined here are always dark, $\left\langle\Phi_{0}|\hat{\mu}| \mathrm{CT}_{n i}^{m j^{\prime}}\right\rangle=0$.

Our zeroth order eigenstates of interest are thus the LE and CT states along with the ground state, $\left|\Phi_{0}\right\rangle$. The only bright states at zeroth order will be the PPP 'plus' Frenkel excitations, $\left|\mathrm{LE}_{n i n j}^{+}\right\rangle$.

\subsection{First order perturbation}

Having obtained zeroth-order eigenstates of $\hat{H}_{0}$, we now consider how they are mixed by the perturbation $\hat{V}$ which occurs when the two monomer are covalently linked, and how this alters the linear absorption spectrum. Following standard perturbation theory, ${ }^{63}$ we form the 'good' degenerate eigenstates of $\hat{H}_{0}$ as linear combinations of excitations which we denote $A$ and $B$ in accordance with the irreducible representations (irreps) of the $C_{2}$ point group. ${ }^{63}$ For Frenkel excitations we have

$$
\begin{aligned}
& \left|\mathrm{LE}_{i j}^{A, \pm}\right\rangle=\frac{1}{\sqrt{2}}\left(\left|\Phi_{n i n j}^{ \pm}\right\rangle+\left|\Phi_{m i m j}^{ \pm}\right\rangle\right), \\
& \left|\mathrm{LE}_{i j}^{B, \pm}\right\rangle=\frac{1}{\sqrt{2}}\left(\left|\Phi_{n i n j}^{ \pm}\right\rangle-\left|\Phi_{m i m j}^{ \pm}\right\rangle\right),
\end{aligned}
$$


and for $\mathrm{CT}$

$$
\begin{aligned}
\left|\mathrm{C} \mathrm{T}_{i j}^{A}\right\rangle & =\frac{1}{\sqrt{2}}\left(\left|\Phi_{n i}^{m j^{\prime}}\right\rangle+\left|\Phi_{m i}^{n j^{\prime}}\right\rangle\right), \\
\left|\mathrm{C} T_{i j}^{B}\right\rangle & =\frac{1}{\sqrt{2}}\left(\left|\Phi_{n i}^{m j^{\prime}}\right\rangle-\left|\Phi_{m i}^{n j^{\prime}}\right\rangle\right),
\end{aligned}
$$

which is similar to the linear combinations used in (for example) Kasha exciton theory, ${ }^{15}$ that considers only the dipole-dipole interactions of Frenkel excitations. Note that in certain cases of degeneracy further linear combinations may be required but we do not find this necessary in what follows.

Since the perturbation $\hat{V}$ is symmetric under all the symmetry operations of the dimer, only like irreps can mix. We are therefore interested in the UV Frenkel excitations $\left|\mathrm{LE}_{14}^{A,+}\right\rangle$ and $\left|\mathrm{LE}_{14}^{B,+}\right\rangle$ mixing with the dark charge-transfer excitations $\left|\mathrm{CT}_{11}^{A}\right\rangle$ and $\left|\mathrm{CT}_{11}^{B}\right\rangle$ respectively. Using standard electronic structure theory algebra ${ }^{69}$ we find

$$
\begin{aligned}
\left\langle\mathrm{LE}_{14}^{A,+}|\hat{V}| \mathrm{CT}_{11}^{A}\right\rangle & =0 \\
\left\langle\mathrm{LE}_{14}^{B,+}|\hat{V}| \mathrm{CT}_{11}^{B}\right\rangle & =\frac{1}{2 \sqrt{2}}\left(t_{n 4^{\prime} m 1^{\prime}}+t_{n 4 m 1}+t_{m 4^{\prime} n 1^{\prime}}+t_{m 4 n 1}\right)
\end{aligned}
$$

where $t_{\text {nimj }}$ is an element of the one-electron, hopping matrix in the molecular orbital basis. Note that due to the NDO approximation present within PPP theory, there are no twoelectron terms in Eq. (9). Equation 8 shows that $\left|\mathrm{CT}_{11}^{A}\right\rangle$ cannot borrow intensity from $\left|\mathrm{LE}_{14}^{A,+}\right\rangle$ (to first order), and we therefore need only consider the corresponding $B$ excitations. This result is particularly convenient for planar or near-planar structures such as 22BP and 22eBP where dipole moment arguments show that only excitations of $B$ symmetry are likely to have significant oscillator strength.

To quantify the extent of mixing, we further simplify the right hand side of Eq. (9). Using the Coulson-Rushbrooke theorem ${ }^{64}$ and the definition of the sign of monomer orbitals (see above) we find that $t_{n 4^{\prime} m 1^{\prime}}=t_{n 4 m 1}$ and $t_{m 4^{\prime} n 1^{\prime}}=t_{m 4 n 1}$. We also find from the $C_{2}$ symmetry 
of the dimer that $t_{n 4^{\prime} m 1^{\prime}}=t_{m 4^{\prime} n 1^{\prime}}$ and $t_{n 4 m 1}=t_{m 4 n 1}$. We can then evaluate the one unique matrix element to obtain

$$
\left\langle\mathrm{LE}_{14}^{B,+}|\hat{V}| \mathrm{CT}_{11}^{B}\right\rangle=\sqrt{2} t_{\nu^{*} \sigma^{*}} c_{n 1, \nu^{*}} c_{m 4, \sigma^{*}}
$$

where $c_{n i}$ represent the expansion coefficients for the monomer orbitals in the atomic orbital basis, $\phi_{n i}=\sum_{\lambda} c_{n i, \lambda} \chi_{\lambda}$. Due to the nearest-neighbor nature of $t$, the only relevant expansion coefficients in Eq. (10) become those associated with the dimer bonding position, i.e., $\nu^{*}$ and $\sigma^{*}$. We further note that $t_{\nu^{*} \sigma^{*}}$ will in general be proportional to $\cos (\theta)$ where $\theta$ is the dihedral angle between the two monomers. Finally, using intensity borrowing perturbation theory, ${ }^{47}$ we find that the dipole moment of the CT excitation is, at first order,

$$
\left\langle\Phi_{0}|\hat{\mu}| \mathrm{CT}_{11}^{B,(1)}\right\rangle \simeq-\left\langle\Phi_{0}|\hat{\mu}| \mathrm{LE}_{14}^{+, B}\right\rangle \frac{\sqrt{2} t_{\nu^{*} \sigma^{*}} c_{n 1, \nu^{*}} c_{m 4, \sigma^{*}}}{E\left(\mathrm{CT}_{11}^{B}\right)-E\left(\mathrm{LE}_{14}^{+, B}\right)}
$$

and the intensity of the new absorption will be proportional to the square of the dipole moment. 63

\section{Application to bipentacenes}

Having arrived at an expression to estimate intensity borrowing to first order in Eq. (11), we undertake an investigation of the extent to which this phenomenon is observed in bipentacenes.

For 11BP a monomer calculation gives $c_{m 4, \sigma^{*}=1}=0$, such that $\left\langle\Phi_{0}|\hat{\mu}| \mathrm{CT}_{11}^{B,(1)}\right\rangle$ and there is no new low-energy absorption. For 22BP a monomer calculation gives $c_{m 4, \sigma^{*}=2} \neq 0$, and we find

$$
\begin{aligned}
-\sqrt{2} t_{0} \cos (\theta) c_{n 1, \nu^{*}=2} c_{m 4, \sigma^{*}=2} & =-0.0860 \mathrm{eV} \\
E\left(\mathrm{CT}_{11}^{B}\right) & =3.58 \mathrm{eV} \\
E\left(\mathrm{LE}_{14}^{B,+}\right) & =3.86 \mathrm{eV}
\end{aligned}
$$


where we use the atom numbering in Fig. 1 and set $t_{0}=2.2 \mathrm{eV}$ and $\theta=37^{\circ}$. Consequently,

$$
\frac{\left\langle\mathrm{LE}_{14}^{B,+}|\hat{V}| \mathrm{CT}_{11}^{B}\right\rangle}{E\left(\mathrm{CT}_{11}^{B}\right)-E\left(\mathrm{LE}_{14}^{+, B}\right)}=0.30 .
$$

The perturbation therefore corresponds to $0.30^{2} \simeq 9 \%$ of the UV peak intensity being borrowed. We emphasize that the $9 \%$ intensity borrowing calculated here is qualitative as we are neglecting all second-order contributions arising from mixing with other states. We further note that although the perturbation is sufficiently significant, leading to visible change in color, is still weak as evidenced by the UV absorption in 22BP and 22eBP continuing to be the dominant absorption. We further calculate the energy of the lowenergy absorption from the first order correction to the energy of the CT state, which is $\left\langle\mathrm{CT}_{11}^{B}|\hat{V}| \mathrm{CT}_{11}^{B}\right\rangle=-\left(n 1 n 1 \mid m 1^{\prime} m 1^{\prime}\right)=-0.91 \mathrm{eV}$, corresponding to the Coulomb attraction of an electron in the LUMO of one molecule and a hole in the HOMO of the other. This gives $E_{\mathrm{CT}_{11}^{B}}^{(1)}=2.67 \mathrm{eV}(464 \mathrm{~nm})$, close to the experimentally observed new transition at $496 \mathrm{~nm}$.

The dihedral angle arguments advanced earlier for the strength of the absorption also explain the more intense absorption seen in 22eBP. Treating the alkene linker to be a molecular 'wire' through which the monomers interact, we estimate the new absorption in $22 \mathrm{eBP}$ to be $\cos ^{2}\left(0^{\circ}\right) / \cos ^{2}\left(37^{\circ}\right) \simeq 1.57$ times greater than the absorption in $22 \mathrm{BP}$. This is experimentally verified by the stronger visible absorption peak that appears in the spectra of Fig. $2 \mathrm{~A}$.

We find that the conclusions reached through intensity borrowing and perturbation theory (Eq. (11)) above can be anticipated by examining the nodal structure of the relevant monomer orbitals. In Fig. 3A, we present the top four HOMOs of TIPS-Pentacene obtained from an RHF calculation that are qualitatively similar to the orbitals from a PPP calculation. The HOMO-3 orbital has nodes on every long-axis carbon (red arrows in Fig. 3A), as is the case for all acenes, ${ }^{8}$ and has been observed experimentally. ${ }^{74}$ The HOMO, HOMO-1 and HOMO-2 all have nodes in the horizontal $(x z)$ plane but the HOMO-3 does not. Now, consider joining together two monomers by the $1,1^{\prime}$ and $2,2^{\prime}$ positions, shown schematically in Fig. 3B. For 

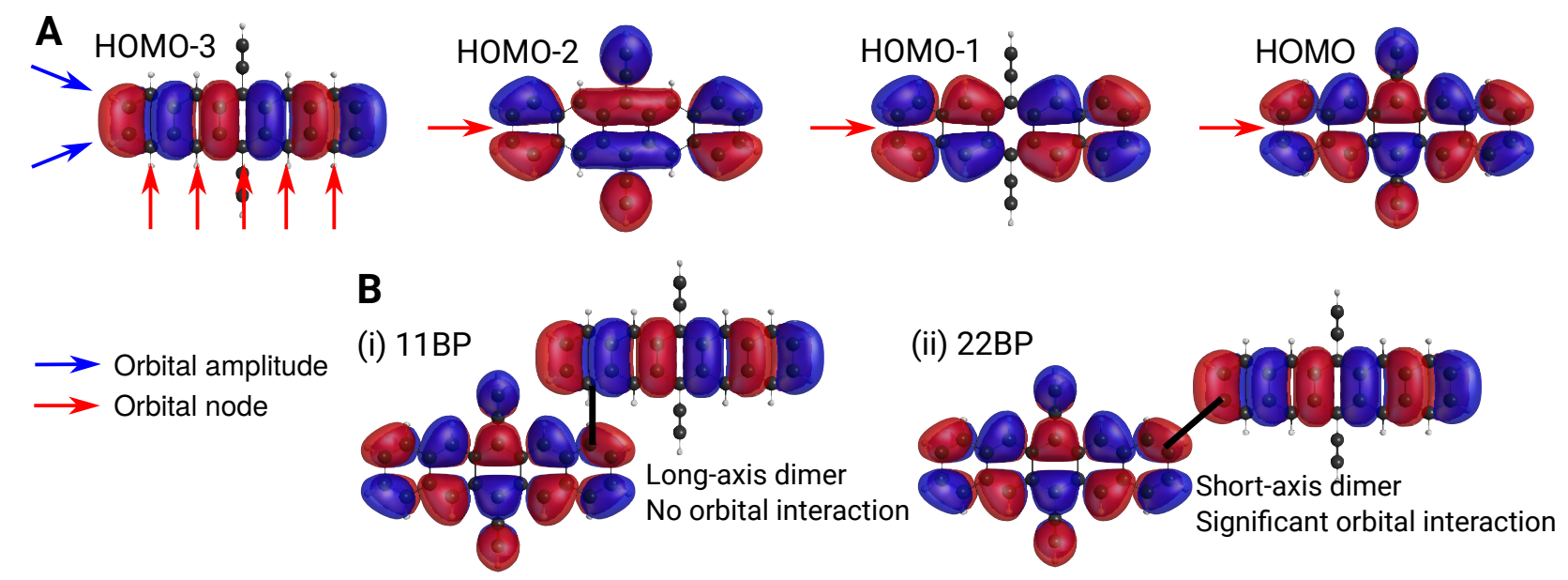

Figure 3: A Top four HOMOs of TIPS-pentacene. Transitions from the HOMO-3 contribute to the intense UV absorption in the monomer and those from the HOMO to a low-energy CT state. B shows how examining nodal structure of orbitals can explain the emergence of an extra absorption. Bonding along a long-axis carbon as for $11 \mathrm{BP}$ in $\mathbf{B}(\mathrm{i})$ leads to no significant interaction between the HOMO-3 on one monomer and the HOMO on the other. Conversely, bonding via a short-axis carbon as for 22BP in $\mathbf{B}$ (ii) leads to a significant orbital interaction. For clarity the dimers are drawn planar and with an elongated inter-monomer bond (thick black line) and long/short axes are defined in Fig. 1A.

11BP in Fig. 3B(i), we see that the HOMO has nonzero amplitude at the 1 position, but the HOMO-3 has zero amplitude such that the perturbation $c_{n 1, \nu^{*}} c_{m 4, \sigma^{*}}=0$, leading to no new low-energy absorption peak in the spectrum. For 22BP, as shown in Fig. 3B(ii), both the HOMO and HOMO-3 have substantial amplitude, $c_{n 1, \nu^{*}} c_{m 4, \sigma^{*}} \neq 0$ and a new low-energy absorption peak is predicted, and verified both by the experimental spectrum and from PPP calculations (Fig. 2).

Using similar arguments, once can show that indirect mixing (via another excitation) is likely to be minimal unless the monomers are joined via a short-axis carbon. The same inferences can be drawn by examining the monomer LUMOs using the Coulson-Rushbrooke theorem, ${ }^{64}$ as shown in the SI.

The intensity borrowing and molecular orbital arguments above show that acene monomers must be joined via short-axis carbons in order to observe a new, low-energy absorption. Since this analysis can, in theory, be performed for arbitrarily many monomers, we would expect this result to hold for oligomers and polymers as well as dimers. In addition, the MO 
arguments suggest that joining acenes via a long-axis carbon or both short-axis carbons will not lead to enhanced low-energy absorption, since the HOMO has a long-axis (xz) nodal plane whereas the HOMO-3 does not, leading to no constructive interaction between the relevant orbitals.

We are now ready to construct a simple design rule to make acene dimers, oligomers and polymers that will exhibit a new low-energy (visible) absorption peak in their spectra:

Join the monomers via a short-axis carbon, and avoid a long-axis symmetry plane passing through adjacent monomers.

This is the main result of the article and is summarized in Fig. 4.

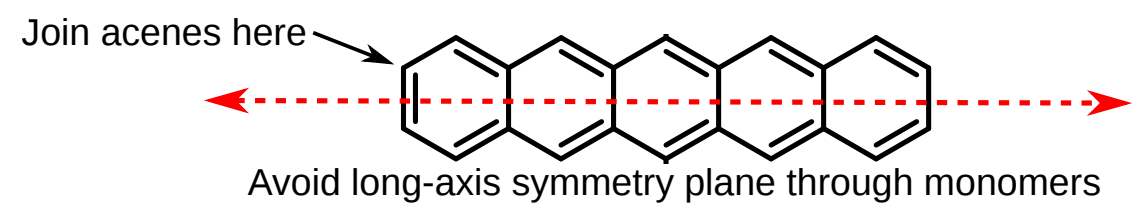

Figure 4: Design rule identifying bonding motifs in acene-based dimers and oligomers that exhibit an additional low-energy absorption

Clearly, for the low-energy absorption to be significant there must be significant interactions between the monomers' $\pi$ systems - they must be directly bonded, otherwise held very close together, or be connected via a conjugated linker (such as in $22 \mathrm{eBP}$ ). Furthermore, if the design rule is satisfied, increasing planarity will increase the intensity of the extra absorption.

\section{$5 \quad$ Application to general acenes}

We have already demonstrated that the design rule proposed in the previous section can explain the presence or absence of a visible absorption peak in the spectra of 11BP, 22BP, and $22 \mathrm{eBP}$. Here, we demonstrate the broad applicability of this rule by anticipating the experimental UV-Vis spectra of acene dimers, trimers, oligomers, and polymers, as well as a variety of hetero-atom substituted derivatives made for a broad range of applications including as a demonstration of organic synthesis, ${ }^{33,34,40,44}$ for organic semiconductors, ${ }^{35,36,38}$ 

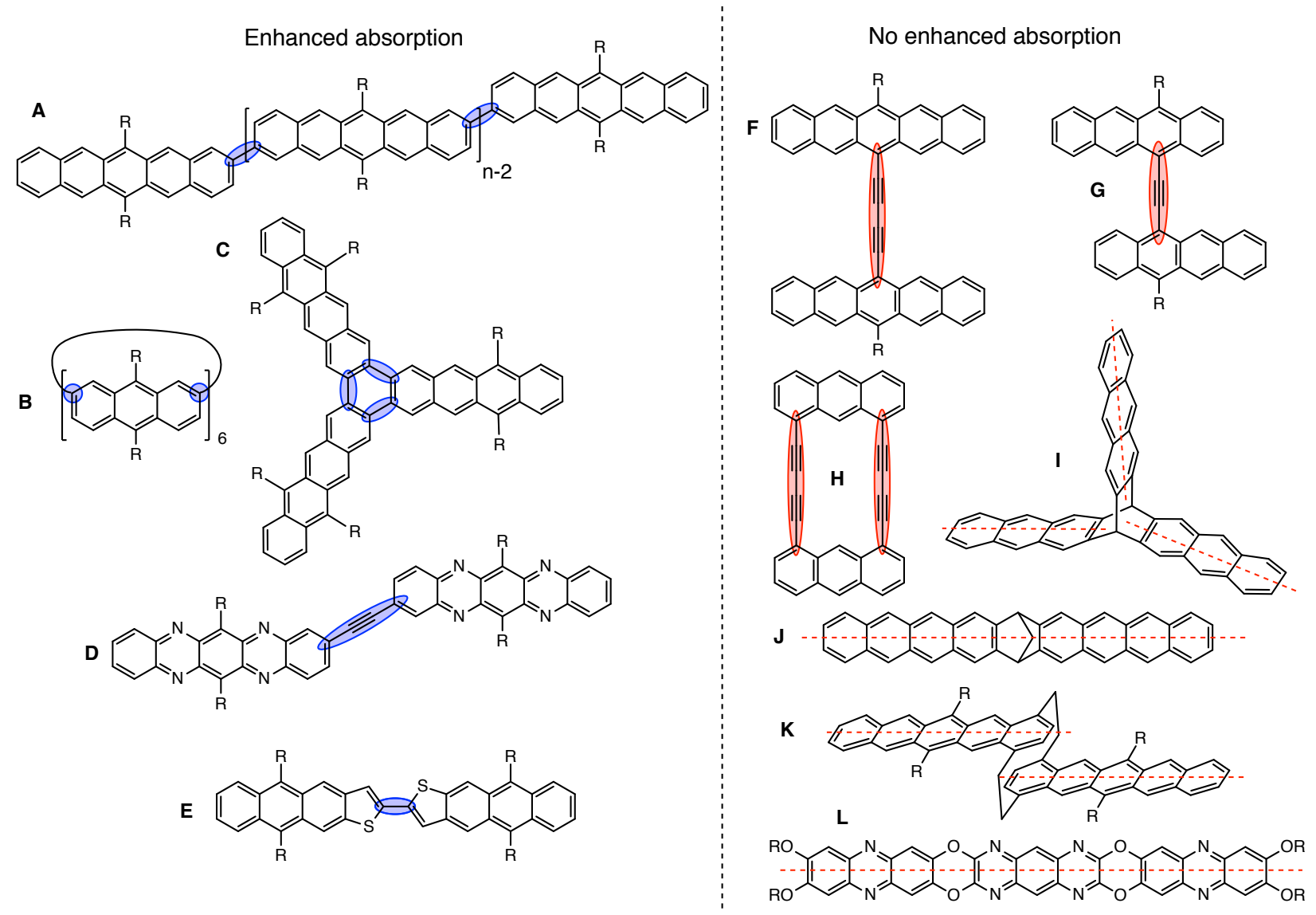

Figure 5: Illustrative examples of acene derivatives, showing how the design rule correctly predicts the presence or absence of an extra, low-energy absorption. Left: molecules bonded via a short-axis carbon (blue oval) and without a horizontal $(x z)$ symmetry plane through adjacent monomers all show enhanced low-energy absorption in their experimental UV-vis spectra. Right: molecules bonded via a long-axis carbon (red oval), or such that the horizontal symmetry plane is preserved through adjacent monomers (red dashes), none of which show enhanced absorption compared to their corresponding monomer(s). The design principle correctly applies to acene dimers, oligomers and polymers such as $\mathrm{A},{ }^{37} \mathrm{~B},{ }^{33} \mathrm{~F},{ }^{38} \mathrm{G}^{39}$ and $\mathrm{H},{ }^{40}$ complex geometries such as $\mathrm{C},{ }^{34} \mathrm{I},{ }^{41,42} \mathrm{~J}^{43}$ and $\mathrm{K},{ }^{44}$ and to heteroatom-substituted dimers $\mathrm{D},{ }^{35} \mathrm{E},{ }^{36}$ and $\mathrm{L} .{ }^{45} \mathrm{R}$ denotes a solubilising or stabilising group.

liquid crystals, ${ }^{41}$ polymer synthesis, ${ }^{42}$ sensors, ${ }^{45}$ photovoltaic applications, ${ }^{37,39,43}$ and one to explore structure-spectrum relationships. ${ }^{32}$

A small selection of these are drawn in Fig. 5 grouped according to whether or not their spectra have enhanced low-energy absorption compared to their corresponding monomers. Our design rule correctly predicts the presence or absence of an extra absorption in all cases, including dimers, oligomers and polymers of naphthalene, ${ }^{32}$ anthracene (Fig. 5B and 
Fig. $5 \mathrm{H}^{33,40}$ ), tetracene (Fig. $5 \mathrm{G}^{39}$ ) and pentacene (Fig. 1, Fig. $5 \mathrm{~A}$ and Fig. 5 $\mathrm{F}^{25,37,38,59}$ ), thereby explaining the structure-spectrum phenomenon first observed in $1948{ }^{32}$ It also correctly predicts the presence or absence of an extra absorption for unusual and com-

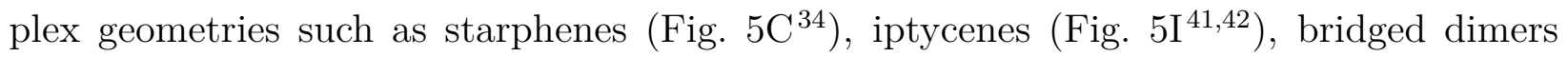
(Fig. $5 \mathrm{~J}^{43}$ ), and cyclophanes (Fig. $5 \mathrm{~K}^{44}$ ). Further, we find this design rule also holds in heteroatom-substituted acene derivatives such as the tetra-aza-pentacene dimer in Fig. 5D, ${ }^{35}$ the anthrathiophene dimer in Fig. 5E $\mathrm{E}^{36}$ and the aza-anthracene trimer Fig. 5L ${ }^{45}$ (compared to its corresponding monomers ${ }^{75,76}$ ). This unexpected applicability of the design rule can be attributed to the 'striped' orbitals in Fig. 3A that will be solutions to the Hückel Hamiltonian of the heteroatom-substituted acene in cases where only the diagonal energies $\left(\epsilon_{\mu}\right)$ are perturbed and not the off-diagonal $\left(t_{\mu \nu}\right)$ terms. In general, this will hold when the the heterosubstitution is only on long-axis carbons. For the thiophene derivative, standard organic chemistry ${ }^{77}$ suggests that the $\mathrm{S}$ heteroatom is of a similar size to a $\mathrm{C}=\mathrm{C}$ double bond, making thiophene qualitatively similar to benzene. With this reasoning the anthrathiophene dimer can be likened to a 2,2'-tetracene dimer, explaining the new low-energy absorption.

Finally, we note that it is challenging to apply the design rule described here to oligomers where two or more moieties overlap in such a way that through-space interaction become important, such as the cross-conjugated dimers in Refs 26 and 78. In these cases a more sophisticated formulation of the hopping term than nearest-neighbor interactions will be required for PPP theory (and the intensity borrowing arguments) to be applied.

\section{Conclusions}

This article uses PPP theory and intensity borrowing perturbation theory to construct a simple rule for predicting and explaining the low-energy absorption spectra of acene derivatives. The resulting testable and experimentally verifiable design rule has been found to hold in a large variety of dimers, trimers, oliogmers and polymers, including those with heteroatom 
substitution and unusual bonding geometries. This is particularly useful because it allows for the presence or absence of a new low-energy absorption to be determined from the monomer orbitals alone, without having to simulate the electronic structure of each dimer or oligomer separately. This a priori design of highly absorbent molecules, of which this is a proof-of-concept, has significant implications for photovoltaic design, where some organic solar photovoltaics are constrained by the small diffusion length of excitons compared to the

thickness of material required to absorb a significant fraction of visible light. ${ }^{22}$ Developing more absorbent molecules that retain the necessary photophysical charge-transport properties could allow for thinner, flexible, cheaper and more efficient solar cells.

\section{Acknowledgement}

We thank Roald Hoffmann and Tao Zeng for helpful discussions and acknowledge spectra from Dan Lehnherr and Rik Tykwinski. We also thank Robert A. DiStasio Jr. and Stuart Althorpe for helpful comments on the manuscript. We thank the Nuckolls lab for use of their UV-vis spectrophotometer. NA acknowledges funding from NSF CAREER grant (Award No. CHE-1555205) and a Sloan Foundation Research Fellowship. TJHH acknowledges funding from NSF EAGER grant (Award No. CHE-1546607) and from Jesus College, Cambridge. This work used the Extreme Science and Engineering Discovery Environment (XSEDE), supported by National Science Foundation grant number ACI-1053575. L.M.C. acknowledges support from the Office of Naval Research Young Investigator Program (Award N00014-151-2532), ACS Petroleum Research Fund, 3M Non-Tenured Faculty Award, Arthur C. Cope Scholar Award, and Cottrell Scholar Award. SNS thanks the NSF for GRFP (DGE 11-44155). This research used resources of the Center for Functional Nanomaterials, which is a U.S. DOE Office of Science Facility, at Brookhaven National Laboratory under Contract No. de-sc0012704. 


\section{References}

(1) Hückel, E. Zur Quantentheorie der Doppelbindung. Zeitschrift für Physik 1930, 60, $423-456$.

(2) Thomas, W. Über die Zahl der Dispersionselektronen, die einem stationären Zustande zugeordnet sind. (Vorläufige Mitteilung). Naturwissenschaften 1925, 13, 627-627.

(3) Reiche, F.; Thomas, W. Über die Zahl der Dispersionselektronen, die einem stationären Zustand zugeordnet sind. Zeitschrift für Physik 1925, 34, 510-525.

(4) Kuhn, W. Über die Gesamtstärke der von einem Zustande ausgehenden Absorptionslinien. Zeitschrift für Physik 1925, 33, 408-412.

(5) Pople, J. A. Electron interaction in unsaturated hydrocarbons. Trans. Faraday Soc. 1953, 49, 1375-1385.

(6) Pople, J. A. The Electronic Spectra of Aromatic Molecules II: A Theoretical Treatment of Excited States of Alternant Hydrocarbon Molecules based on Self-Consistent Molecular Orbitals. P. Phys. Soc. Lond.. Section A 1955, 68, 81.

(7) Pariser, R.; Parr, R. G. A Semi-Empirical Theory of the Electronic Spectra and Electronic Structure of Complex Unsaturated Molecules. I. J. Chem. Phys. 1953, 21, 466-471.

(8) Pariser, R. Theory of the Electronic Spectra and Structure of the Polyacenes and of Alternant Hydrocarbons. J. Chem. Phys. 1956, 24, 250-268.

(9) Zeng, T.; Hoffmann, R.; Ananth, N. The Low-Lying Electronic States of Pentacene and Their Roles in Singlet Fission. J. Am. Chem. Soc. 2014, 136, 5755-5764.

(10) Hajgató, B.; Szieberth, D.; Geerlings, P.; De Proft, F.; Deleuze, M. S. A benchmark theoretical study of the electronic ground state and of the singlet-triplet split of benzene and linear acenes. J. Chem. Phys. 2009, 131. 
(11) Casanova, D.; Head-Gordon, M. Restricted active space spin-flip configuration interaction approach: theory, implementation and examples. Phys Chem Chem Phys 2009, 11, 9779-90.

(12) Sony, P.; Shukla, A. Large-scale correlated calculations of linear optical absorption and low-lying excited states of polyacenes: Pariser-Parr-Pople Hamiltonian. Phys. Rev. B 2007, 75, 155208.

(13) Hashimoto, T.; Nakano, H.; Hirao, K. Theoretical study of the valence $\pi \rightarrow \pi^{*}$ excited states of polyacenes: Benzene and naphthalene. J. Chem. Phys. 1996, 104, 6244-6258.

(14) Kawashima, Y.; Hashimoto, T.; Nakano, H.; Hirao, K. Theoretical study of the valence $\pi \rightarrow \pi^{*}$ excited states of polyacenes: anthracene and naphthacene. Theor. Chem. Acc. 1999, 102, 49-64.

(15) M. Kasha, H. R. R.; El-Bayoumi, M. A. The exciton model in molecular spectroscopy. Pure Appl. Chem. 1965, 11, 371-392.

(16) Kazmaier, P. M.; Hoffmann, R. A Theoretical Study of Crystallochromy. Quantum Interference Effects in the Spectra of Perylene Pigments. J. Am. Chem. Soc. 1994, 116, 9684-9691.

(17) Hanson, K.; Roskop, L.; Djurovich, P. I.; Zahariev, F.; Gordon, M. S.; Thompson, M. E. A Paradigm for Blue- or Red-Shifted Absorption of Small Molecules Depending on the Site of $\pi$-Extension. J. Am. Chem. Soc. 2010, 132, 16247-16255.

(18) Mishra, A.; Fischer, M.; Bäuerle, P. Metal-Free Organic Dyes for Dye-Sensitized Solar Cells: From Structure: Property Relationships to Design Rules. Angew. Chem., Int. Ed. 2009, 48, 2474-2499.

(19) Zollinger, H. Color chemistry: syntheses, properties, and applications of organic dyes and pigments; John Wiley \& Sons, 2003. 
(20) Christie, R. Colour Chemistry; Royal Society of Chemistry: Cambridge, UK, 2015.

(21) Kippelen, B.; Bredas, J.-L. Organic photovoltaics. Energy Environ. Sci. 2009, 2, 251261.

(22) Forrest, S. R. The Limits to Organic Photovoltaic Cell Efficiency. MRS Bulletin 2005, $30,28-32$.

(23) Chan, W.-L.; Ligges, M.; Jailaubekov, A.; Kaake, L.; Miaja-Avila, L.; Zhu, X.-Y. Observing the Multiexciton State in Singlet Fission and Ensuing Ultrafast Multielectron Transfer. Science 2011, 334, 1541-1545.

(24) Walker, B. J.; Musser, A. J.; Beljonne, D.; Friend, R. H. Singlet exciton fission in solution. Nat. Chem. 2013, 5, 1019-1024.

(25) Sanders, S. N.; Kumarasamy, E.; Pun, A. B.; Trinh, M. T.; Choi, B.; Xia, J.; Taffet, E. J.; Low, J. Z.; Miller, J. R.; Roy, X. et al. Quantitative Intramolecular Singlet Fission in Bipentacenes. J. Am. Chem. Soc. 2015, 137, 8965-72.

(26) Zirzlmeier, J.; Lehnherr, D.; Coto, P. B.; Chernick, E. T.; Casillas, R.; Basel, B. S.; Thoss, M.; Tykwinski, R. R.; Guldi, D. M. Singlet fission in pentacene dimers. Proc. Natl. Acad. Sci. U.S.A. 2015, 112, 5325-30.

(27) Smith, M. B.; Michl, J. Singlet Fission. Chem. Rev. 2010, 110, 6891-6936.

(28) Smith, M. B.; Michl, J. Recent advances in singlet fission. Annu Rev Phys Chem 2013, $64,361-86$.

(29) Pun, A. B.; Sanders, S. N.; Kumarasamy, E.; Sfeir, M. Y.; Congreve, D. N.; Campos, L. M. Triplet Harvesting from Intramolecular Singlet Fission in Polytetracene. Advanced Materials 2017, 29, 1701416-n/a, 1701416. 
(30) Sanders, S. N.; Kumarasamy, E.; Pun, A. B.; Steigerwald, M. L.; Sfeir, M. Y.; Campos, L. M. Intramolecular Singlet Fission in Oligoacene Heterodimers. Angew. Chem., Int. Ed. 2016, 55, 3373-7.

(31) Lukman, S.; Musser, A. J.; Chen, K.; Athanasopoulos, S.; Yong, C. K.; Zeng, Z.; Ye, Q.; Chi, C.; Hodgkiss, J. M.; Wu, J. et al. Tuneable Singlet Exciton Fission and Triplet-Triplet Annihilation in an Orthogonal Pentacene Dimer. Adv. Funct. Mater. 2015, 25, 5452-5461.

(32) Friedel, R. A.; Orchin, M.; Reggel, L. Steric Hindrance and Short Wave Length Bands in the Ultraviolet Spectra of Some Naphthalene and Diphenyl Derivatives. J. Am. Chem. Soc. 1948, 70, 199-204.

(33) Yamamoto, Y.; Wakamatsu, K.; Iwanaga, T.; Sato, H.; Toyota, S. Macrocyclic 2,7Anthrylene Oligomers. Chem.-Asian J. 2016, 11, 1370-1375.

(34) Rüdiger, E. C.; Porz, M.; Schaffroth, M.; Rominger, F.; Bunz, U. H. F. Synthesis of soluble, alkyne-substituted trideca- and hexadeca-starphenes. Chemistry 2014, 20, $12725-8$.

(35) Geyer, F. L.; Brosius, V.; Bunz, U. H. F. 2-Bromotetraazapentacene and Its Functionalization by $\operatorname{Pd}(0)$-Chemistry. J. Org. Chem. 2015, 80, 12166-76.

(36) Balandier, J.-Y.; Quist, F.; Stas, S.; Tylleman, B.; Ragoen, C.; Mayence, A.; Bouzakraoui, S.; Cornil, J.; Geerts, Y. H. Dimers of Anthrathiophene and Anthradithiophene Derivatives: Synthesis and Characterization. Org. Lett. 2011, 13, 548-551.

(37) Kumarasamy, E.; Sanders, S. N.; Pun, A. B.; Vaselabadi, S. A.; Low, J. Z.; Sfeir, M. Y.; Steigerwald, M. L.; Stein, G. E.; Campos, L. M. Properties of Poly- and Oligopentacenes Synthesized from Modular Building Blocks. Macromolecules 2016, 49, 1279-1285. 
(38) Lehnherr, D.; Murray, A.; McDonald, R.; Tykwinski, R. A Modular Synthetic Approach to Conjugated Pentacene Di-, Tri-, and Tetramers. Angew. Chem., Int. Ed. 2010, 49, 6190-6194.

(39) Barlier, V. S.; Schlenker, C. W.; Chin, S. W.; Thompson, M. E. Acetylide-bridged tetracene dimers. Chem. Commun. (Camb) 2011, 47,3754-6.

(40) Akiyama, S.; Misumi, S.; Nakagawa, M. Cyclic Acetylenes. IV. Synthesis of a Cyclic Tetraacetylene Containing Anthracene Nuclei. B. Chem. Soc. Jpn. 1960, 33, 1293-1298.

(41) Long, T. M.; Swager, T. M. Minimization of Free Volume: Alignment of Triptycenes in Liquid Crystals and Stretched Polymers. Adv. Mater. 2001, 13, 601-604.

(42) Bhola, R.; Payamyar, P.; Murray, D. J.; Kumar, B.; Teator, A. J.; Schmidt, M. U.; Hammer, S. M.; Saha, A.; Sakamoto, J.; Schlüter, A. D. et al. A Two-Dimensional Polymer from the Anthracene Dimer and Triptycene Motifs. J. Am. Chem. Soc. 2013, $135,14134-14141$.

(43) Cook, J. D.; Carey, T. J.; Damrauer, N. H. Solution-Phase Singlet Fission in a Structurally Well-Defined Norbornyl-Bridged Tetracene Dimer. J. Phys. Chem. A 2016, 120, 4473.

(44) Bula, R.; Fingerle, M.; Ruff, A.; Speiser, B.; Maichle-Mössmer, C.; Bettinger, H. F. Anti -[2.2](1,4)pentacenophane: A Covalently Coupled Pentacene Dimer. Angew. Chem., Int. Ed. 2013, 52, 11647-11650.

(45) Gu, P.-Y.; Gao, J.; Wang, C.; Zhang, Q. Synthesis, physical properties and ion recognition of a novel larger heteroacene with eleven linearly-fused rings and two different types of heteroatom. RSC Adv. 2015, 5, 80307-80310.

(46) Zheng, L.; Polizzi, N. F.; Dave, A. R.; Migliore, A.; Beratan, D. N. Where Is the Elec- 
tronic Oscillator Strength? Mapping Oscillator Strength across Molecular Absorption Spectra. J. Phys. Chem. A 2016, 120, 1933-1943.

(47) Robinson, G. W. Intensity Enhancement of Forbidden Electronic Transitions by Weak Intermolecular Interactions. J. Chem. Phys. 1967, 46, 572.

(48) Coulson, C. A. Excited Electronic Levels in Conjugated Molecules: I. Long Wavelength Ultra-Violet Absorption of Naphthalene, Anthracene and Homologs. P. Phys. Soc. Lond. 1948, 60, 257.

(49) Dewar, M. J. S.; Longuet-Higgins, H. C. The Electronic Spectra of Aromatic Molecules I: Benzenoid Hydrocarbons. P. Phys. Soc. Lond.. Section A 1954, 67, 795.

(50) Akiyama, S.; Nakagawa, M. Cyclic Acetylenes. III. Synthesis of 1,1'-Dianthryldiacetylene. A Model Substance of a Cyclic Tetraacetylene Containing Anthracene Nuclei. B. Chem. Soc. Jpn. 1960, 33, 1291-1293.

(51) Tanaka, J. The Electronic Spectra of Pyrene, Chrysene, Azulene, Coronene and Tetracene Crystals. B. Chem. Soc. Jpn. 1965, 38, 86-102.

(52) Clar, E.; schmidt, W. Localised vs delocalised molecular orbitals in aromatic hydrocarbons. Tetrahedron 1979, 35, $2673-2680$.

(53) Halasinski, T. M.; Hudgins, D. M.; Salama, F.; Allamandola, L. J.; Bally, T. Electronic Absorption Spectra of Neutral Pentacene $\left(\mathrm{C}_{22} \mathrm{H}_{14}\right)$ and Its Positive and Negative Ions in Ne, Ar, and Kr Matrices. J. Phys. Chem. A 2000, 104, 7484-7491.

(54) Payne, M. M.; Parkin, S. R.; Anthony, J. E. Functionalized Higher Acenes: Hexacene and Heptacene. J. Am. Chem. Soc. 2005, 127, 8028-8029, PMID: 15926823.

(55) Lehnherr, D.; Gao, J.; Hegmann, F. A.; Tykwinski, R. R. Synthesis and Electronic Properties of Conjugated Pentacene Dimers. Org. Lett. 2008, 10, 4779-4782. 
(56) Yamagata, H.; Norton, J.; Hontz, E.; Olivier, Y.; Beljonne, D.; BrÃldas, J. L.; Silbey, R. J.; Spano, F. C. The nature of singlet excitons in oligoacene molecular crystals. J. Chem. Phys. 2011, 134, 204703.

(57) Beljonne, D.; Yamagata, H.; Brédas, J. L.; Spano, F. C.; Olivier, Y. Charge-transfer excitations steer the Davydov splitting and mediate singlet exciton fission in pentacene. Phys. Rev. Lett. 2013, 110, 226402.

(58) Kurashige, Y.; Yanai, T. Theoretical Study of the $\pi \rightarrow \pi^{*}$ Excited States of Oligoacenes: A Full $\pi$-Valence DMRG-CASPT2 Study. B. Chem. Soc. Jpn. 2014, 87, 1071-1073.

(59) Fuemmeler, E. G.; Sanders, S. N.; Pun, A. B.; Kumarasamy, E.; Zeng, T.; Miyata, K.; Steigerwald, M. L.; Zhu, X.-Y.; Sfeir, M. Y.; Campos, L. M. et al. A Direct Mechanism of Ultrafast Intramolecular Singlet Fission in Pentacene Dimers. ACS Cent. Sci. 2016, 2, 316-324.

(60) Suzuki, S.; Fujii, T.; Baba, H. Interpretation of electronic spectra by configuration analysis. J. Mol. Spectrosc. 1973, 47, 243-251.

(61) Koutecký, J. Possible reinterpretation of the Pariser-Parr-Pople theory. Chem. Phys. Lett. 1967, 1, $249-250$.

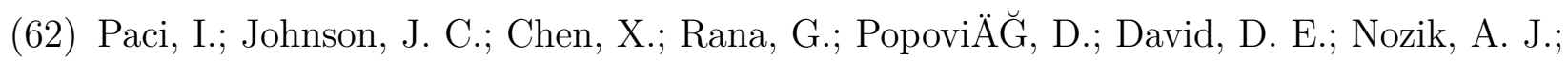
Ratner, M. A.; Michl, J. Singlet Fission for Dye-Sensitized Solar Cells: Can a Suitable Sensitizer Be Found? Journal of the American Chemical Society 2006, 128, 16546-16553, PMID: 17177404.

(63) Atkins, P. W.; Friedman, R. S. Molecular quantum mechanics; Oxford University Press, 2011.

(64) Coulson, C. A.; Rushbrooke, G. S. Note on the method of molecular orbitals. Math. Proc. Cambridge 1940, 36, 193-200. 
(65) Cook, J. D.; Carey, T. J.; Arias, D. H.; Johnson, J. C.; Damrauer, N. H. SolventControlled Branching of Localized versus Delocalized Singlet Exciton States and Equilibration with Charge Transfer in a Structurally Well-Defined Tetracene Dimer. J. Phys. Chem. A 2017, 121, 9229-9242, PMID: 29160072.

(66) Kumarasamy, E.; Sanders, S. N.; Tayebjee, M. J. Y.; Asadpoordarvish, A.; Hele, T. J. H.; Fuemmeler, E. G.; Pun, A. B.; Yablon, L. M.; Low, J. Z.; Paley, D. W. et al. Tuning Singlet Fission in Homoconjugated Pi-Bridge-Pi Dimers. J. Am. Chem. Soc. 2017, 139, 12488-12494.

(67) Jeziorski, B.; Moszynski, R.; Szalewicz, K. Perturbation Theory Approach to Intermolecular Potential Energy Surfaces of van der Waals Complexes. Chemical Reviews 1994, 94, 1887-1930.

(68) Aryanpour, K.; Muñoz, J. A.; Mazumdar, S. Does Singlet Fission Enhance the Performance of Organic Solar Cells? J. Phys. Chem. C 2013, 117, 4971-4979.

(69) Szabo, A.; Ostlund, N. Modern Quantum Chemistry: Introduction to Advanced Electronic Structure Theory; Dover Books on Chemistry; Dover Publications, 1989.

(70) Li, X.; Parrish, R. M.; Liu, F.; Kokkila Schumacher, S. I. L.; Martínez, T. J. An Ab Initio Exciton Model Including Charge-Transfer Excited States. Journal of Chemical Theory and Computation 2017, 13, 3493-3504, PMID: 28617595.

(71) Mulliken, R. S. Intensities of Electronic Transitions in Molecular Spectra II. ChargeTransfer Spectra. J. Chem. Phys. 1939, 7, 20-34.

(72) Feng, X.; Luzanov, A. V.; Krylov, A. I. Fission of Entangled Spins: An Electronic Structure Perspective. J. Phys. Chem. Lett. 2013, 4, 3845-3852.

(73) Pieniazek, P. A.; Krylov, A. I.; Bradforth, S. E. Electronic structure of the benzene dimer cation. J. Chem. Phys. 2007, 127, 044317. 
(74) Soe, W.-H.; Manzano, C.; De Sarkar, A.; Chandrasekhar, N.; Joachim, C. Direct Observation of Molecular Orbitals of Pentacene Physisorbed on $\mathrm{Au}(111)$ by Scanning Tunneling Microscope. Phys. Rev. Lett. 2009, 102, 176102.

(75) Lee, D.-C.; Cao, B.; Jang, K.; Forster, P. M. Self-assembly of halogen substituted phenazines. J. Mater. Chem. 2010, 20, 867-873.

(76) Gawrys, P.; Marszalek, T.; Bartnik, E.; Kucinska, M.; Ulanski, J.; Zagorska, M. Novel, Low-Cost, Highly Soluble n-Type Semiconductors: Tetraazaanthracene Tetraesters. Org. Lett. 2011, 13, 6090-6093.

(77) Clayden, J.; Greeves, N.; Warren, S.; Wothers, P. Organic chemistry; Oxford University Press, 2001.

(78) Zirzlmeier, J.; Casillas, R.; Reddy, S. R.; Coto, P. B.; Lehnherr, D.; Chernick, E. T.; Papadopoulos, I.; Thoss, M.; Tykwinski, R. R.; Guldi, D. M. Solution-based intramolecular singlet fission in cross-conjugated pentacene dimers. Nanoscale 2016, 8, 10113-23. 


\title{
Supporting information for:
}

\section{Anticipating acene-based chromophore spectra with molecular orbital arguments}

\author{
Timothy J. H. Hele, ${ }^{1, *}$ Eric G. Fuemmeler, ${ }^{2}$ Samuel N. Sanders, ${ }^{3}$ Elango \\ Kumarasamy, ${ }^{3}$ Matthew Y. Sfeir, ${ }^{4}$ Luis M. Campos, ${ }^{3, \dagger}$ and Nandini Ananth ${ }^{2, \ddagger}$ \\ ${ }^{1}$ Cavendish Laboratory, JJ Thomson Avenue, \\ Cambridge University, CB3 OHE, UK \\ ${ }^{2}$ Baker Laboratory, 259 East Avenue, Cornell University, Ithaca, NY 14850, USA \\ ${ }^{3}$ Department of Chemistry, Columbia University, New York, NY 10027, USA \\ ${ }^{4}$ Center for Functional Nanomaterials, \\ Brookhaven National Laboratory, Upton, NY 11973, USA
}

(Dated: December 20, 2018)

\footnotetext{
*tjhh2@cam.ac.uk

† lcampos@columbia.edu

‡ananth@cornell.edu
} 


\section{CONTENTS}

I. Computational details $\quad 3$

II. Supplementary Figures $\quad 5$

$\begin{array}{ll}\text { III. Experimental methods } & 7\end{array}$

$\begin{array}{ll}\text { A. General Methods } & 7\end{array}$

$\begin{array}{ll}\text { B. General protocol for the synthesis of bipentacenes } & 7\end{array}$

C. General protocol for the synthesis of trans-ethylene-2,2-bipentacene 9

D. General procedure for the synthesis of BPin derivatives of pentacenes 9

$\begin{array}{ll}\text { 1. Molecule } 3 & 9\end{array}$

2. Molecule $4 \quad 10$

3. Molecule $5 \quad 11$

E. Synthesis of bipentacenes: 12

1. $11 \mathrm{BP} \quad 12$

2. $22 \mathrm{eBP} \quad 13$

IV. Co-ordinates for electronic structure calculations 14

A. TIPS-pentacene 14

$\begin{array}{ll}\text { B. } 11 \mathrm{BP} & 15\end{array}$

$\begin{array}{ll}\text { C. } 22 \mathrm{BP} & 18\end{array}$

$\begin{array}{ll}\text { D. } 22 \mathrm{eBP} & 18\end{array}$

E. $66 y B P \quad 21$

V. NMR data $\quad 25$

References 33 


\section{COMPUTATIONAL DETAILS}

All calculations were performed in vacuo as the ground states of alternant hydrocarbons such as pentacene have no dipole (or multipole) and are therefore not expected to show a solvatochromic shift.

Our first task is to compute the ground-state structures of these molecules. Consistent with previous calculations on TIPS-pentacene derivatives, we convert the $\operatorname{Si}(i \text {-Pr })_{3}$ groups to hydrogens as they do not significantly affect the electronic structure of the chromophore [1]. Similar to previous calculations on the pentacene monomer (without the TIPS groups) [2], we optimize the monomer structure using complete active-space self-consistent field (CASSCF), here at the 8o8e level with orbitals chosen by energy (four highest HOMOs and four lowest LUMOs), using a restricted Hartree-Fock (RHF) reference and no state-averaging. As in previous work [2] we used a trimmed cc-pVTZ basis, with the $f$ functions removed from carbon and the $d$ functions from hydrogen. The 22BP structure was taken from Ref. 1. Due to computational cost, 11BP, $22 \mathrm{eBP}$, and $66 \mathrm{yBP}$ were optimized using DFT with the B3LYP functional in a $6-31 \mathrm{G}^{*}$ basis. Coordinates are given in section IV of the SI.

High-level monomer calculations used CASSCF and generalized multiconfiguational quasidegenerate perturbation theory (GMC-QDPT) with the same basis as for optimization. CASSCF calculations were state-averaged over the lowest three states of a given symmetry, and GMC-QDPT calculations used the standard intruder state avoidance parameter of $0.02 E_{h}^{2}$ and Granovsky's zeroth-order Hamiltonian for XMC-QDPT [3]. Pictures of monomer MOs were generated using the same basis from a restricted Hartree-Fock (RHF) calculation, have the same qualitative structure as from a PPP calculation, and are ordered according to their PPP energy.

Similar to previous research [4] for PPP calculations the hopping parameter $t_{\mu \nu}$ was set to $2.2 \mathrm{eV}$ for single bonds, $2.4 \mathrm{eV}$ for aromatic bonds, and $2.8 \mathrm{eV}$ for triple bonds, scaled by a cosine of the dihedral angle for inter-monomer bonds [5]. The electronic repulsion terms

were parameterized using the Mataga-Nishimoto form [6]: $V_{\mu \nu}=U /\left(1+\frac{R_{\mu \nu}}{r_{0}}\right)$, where the Hubbard parameter $U=8 \mathrm{eV}[4]$ and $r_{0}=1.634 \AA$, which was found to give good agreement for the pentacene monomer (see Table I below). For highly non-planar molecules, other 


\begin{tabular}{c|c|c|c|c|c|c}
$\begin{array}{c}\text { Exp. abs. } \\
\lambda(\mathrm{nm})\end{array}$ & $\begin{array}{c}\text { GMC-QDPT } \\
\text { state }\end{array}$ & $\begin{array}{c}\text { GMC-QDPT } \\
\text { excitations }\end{array}$ & $\begin{array}{c}\text { GMC-QDPT } \\
\lambda(\mathrm{nm})\end{array}$ & PPP state & PPP excitations & $\begin{array}{c}\text { PPP } \\
\text { (n) }\end{array}$ \\
\hline $1 B_{2 u}$ & $0.94\left|\Phi_{1}^{1^{\prime}}\right\rangle$ & 597 & $1 B_{2 u}$ & $0.99 \mid \Phi_{1}^{\left.1^{\prime}\right\rangle}$ & 662 \\
440 & $1 B_{3 u}$ & $0.72\left|\Phi_{4}^{1^{\prime}}\right\rangle-0.58\left|\Phi_{1}^{4^{\prime}}\right\rangle$ & 460 & $1 B_{3 u}$ & $0.67\left(\left|\Phi_{4}^{1^{\prime}}\right\rangle-\left|\Phi_{1}^{4^{\prime}}\right\rangle\right)$ & 385 \\
310 & $3 B_{3 u}$ & $0.59\left|\Phi_{4}^{1^{\prime}}\right\rangle+0.71\left|\Phi_{1}^{4^{\prime}}\right\rangle$ & 307 & $2 B_{3 u}$ & $0.69\left(\left|\Phi_{4}^{1^{\prime}}\right\rangle+\left|\Phi_{1}^{4^{\prime}}\right\rangle\right)$ & 317
\end{tabular}

TABLE I. Energies of selected excited states of TIPS-pentacene computed using 8o8e CASSCF with GMC-QDPT, and PPP theory (see computational details). PPP orbital numbering is used [12], where $\left|\Phi_{i}^{j^{\prime}}\right\rangle$ is a singlet spin-adapted excitation from monomer orbital $i$ to monomer orbital $j^{\prime}$. For comparison, orbitals are ordered according to their PPP energy, and their signs are chosen to be consistent with the Coulson-Rushbrooke theorem $[12,13]$. The GMC-QDPT $2 B_{3 u}$ state is predominantly composed of double excitations and is therefore not captured by PPP theory.

NDO methods such as INDO could be used for spectral prediction [7, 8] but conventional PPP theory is found to be sufficiently accurate for the molecules studied (see Fig. 2 of the main article). For plotting the results of PPP calculations, the excited state energies were not shifted to agree with experiment. Peaks were broadened used a full width at half maximum value of $20 \mathrm{~nm}$, and do not include vibrational progressions. PPP calculations were performed using in-house code finding the lowest 25 singly excited states. All other electronic structure calculations were performed using the GAMESS-US package [9, 10], using the standard input parameters unless otherwise stated, and used XSEDE resources [11]. Orbitals are plotted using Macmolplt and a contour value of 0.01 .

In Table I we compare the results of GMC-QDPT, PPP theory and experiment for relevant low-lying excited states.

Examining the high-level and PPP calculations in Table I, we find that PPP is more accurate than GMC-QDPT for the lowest-energy transition, less accurate for the dark $1 B_{3 u}$ state and both methods capture the UV excitation around 310nm to within 10nm. Importantly, the excitations corresponding to the excited states in Table I are the same in both GMCQDPT and PPP and weighted by similar coefficients, showing that despite the simplicity of PPP theory, it provides an accurate description of these excited states. 


\section{SUPPLEMENTARY FIGURES}
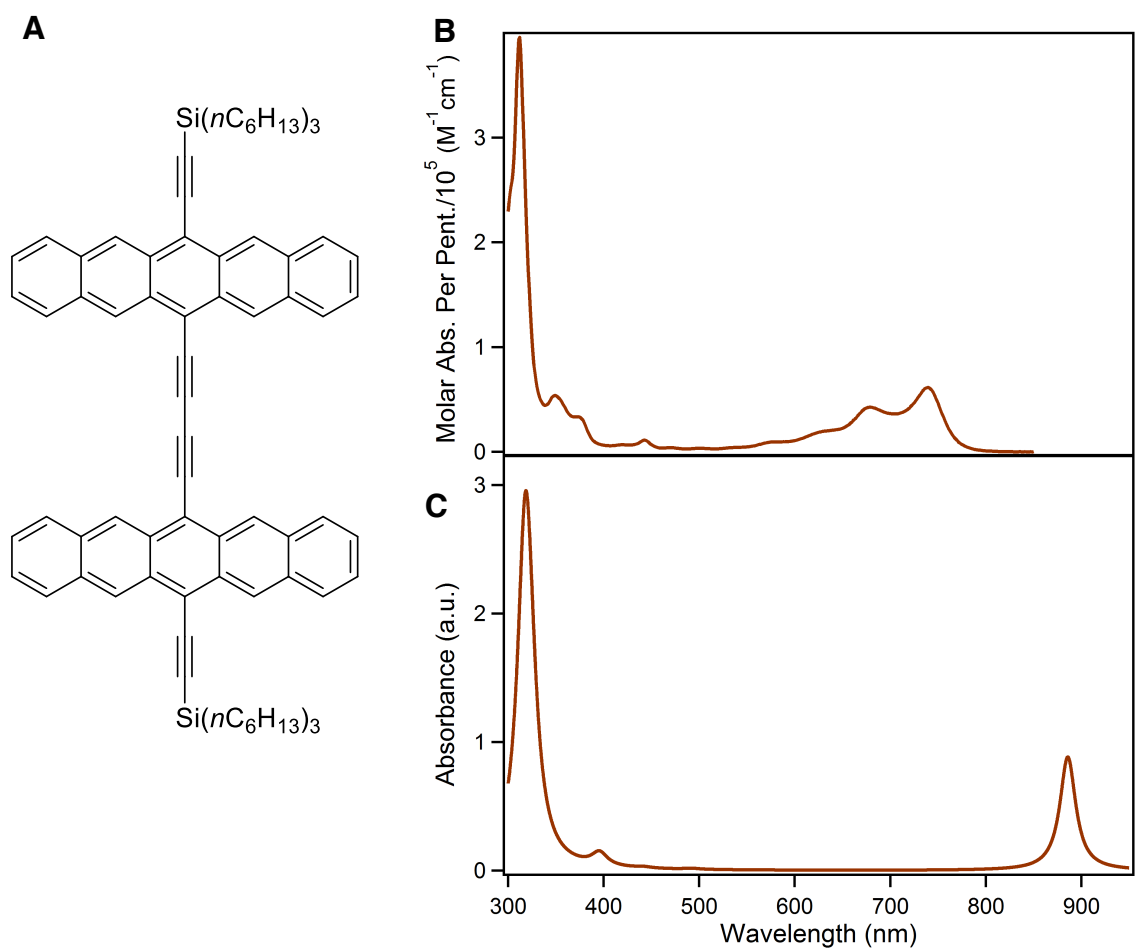

FIG. 1. Structure and spectra of the planar bipentacene 66yBP. (A), Chemical structure of the alkyne-linked 6,6'-bipentacene [14]. Neither the experimental spectrum [14] (B) nor computed spectrum using PPP theory $(\mathbf{C})$ show an extra low-energy absorption compared to TIPS-P. This is despite the ability of the dimer to lie flat, and the large inter-chromophore interaction which causes the lowest-energy excitation to be redshifted by approximately $100 \mathrm{~nm}$ compared to monomeric TIPS-Pentacene. 


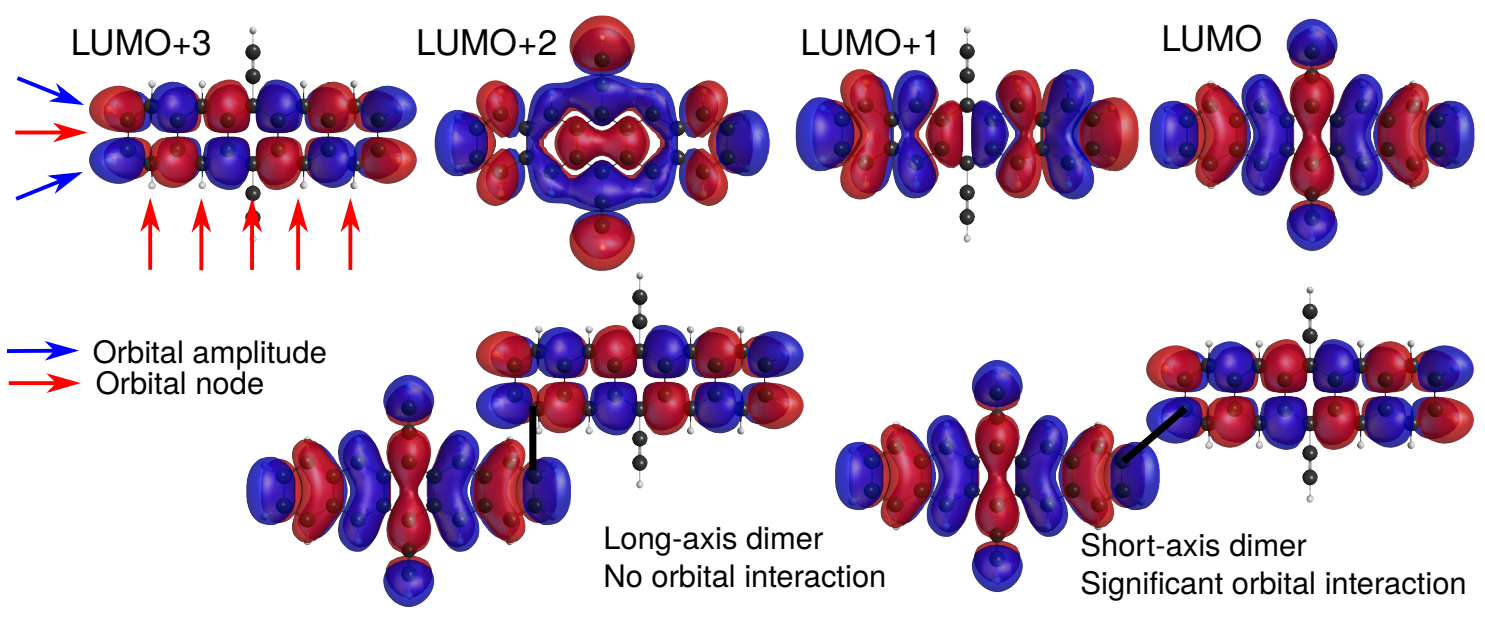

FIG. 2. Monomer LUMOs. LUMOs of TIPS-pentacene, showing how the LUMO+3, like the HOMO-3, has nodes on all long-axis tertiary carbons. Furthermore, the LUMO+3 has a node in the long-axis $(x z)$ symmetry plane, whereas none of the other LUMOs shown do. 


\section{EXPERIMENTAL METHODS}

\section{A. General Methods}

All commercially obtained reagents/solvents were used as received; chemicals were purchased from Alfa Aesar ${ }^{\circledR}$, Sigma-Aldrich ${ }^{\circledR}$, Acros organics ${ }^{\circledR}$, TCI America ${ }^{\circledR}$, Mallinckrodt ${ }^{\circledR}$, and Oakwood ${ }^{\circledR}$ Products, and were used as received without further purification. Unless stated otherwise, reactions were conducted in oven-dried glassware under argon atmosphere. ${ }^{1} \mathrm{H}-\mathrm{NMR}$ and ${ }^{13} \mathrm{C}-\mathrm{NMR}$ spectra were recorded on Bruker $400 \mathrm{MHz}\left(100 \mathrm{MHz}\right.$ for $\left.{ }^{13} \mathrm{C}\right)$ and on $500 \mathrm{MHz}\left(125 \mathrm{MHz}\right.$ for $\left.{ }^{13} \mathrm{C}\right)$ spectrometers. Data from the ${ }^{1} \mathrm{H}-\mathrm{NMR}$ and ${ }^{13} \mathrm{C}$ spectroscopy are reported as chemical shift $(\delta \mathrm{ppm})$ with the corresponding integration values. Coupling constants $(J)$ are reported in hertz $(\mathrm{Hz})$. Standard abbreviations indicating multiplicity were used as follows: s (singlet), b (broad), d (doublet), t (triplet), q (quartet), m (multiplet) and virt (virtual). The mass spectral data for the compounds were obtained from XEVO G2-XS Waters ${ }^{\circledR}$ equipped with a QTOF detector with multiple inlet and ionization capabilities including electrospray ionization (ESI), atmospheric pressure chemical ionization (APCI), and atmospheric solids analysis probe (ASAP). The base peaks were usually obtained as $[\mathrm{M}]^{+}$or $[\mathrm{M}+\mathrm{H}]^{+}$ions.

Anhydrous solvents were obtained from a Schlenk manifold with purification columns packed with activated alumina and supported copper catalyst (Glass Contour, Irvine, CA). All reactions were carried out under argon unless otherwise noted.

\section{B. General protocol for the synthesis of bipentacenes}

The general synthetic scheme is similar to the previously-reported synthesis of $22 \mathrm{BP}[1$, 15, 16]. For more details on the synthesis of 2,2-BP and other intermediates please refer to our previous publications $[1,15,16]$. 
Synthesis of Bpin derivatives of TIPS pentacenes:<smiles>BC(Br)c1cccc(Br)c1C(Br)Br</smiles><smiles>O=C1C=CC(=O)c2cc3ccccc3cc21</smiles>

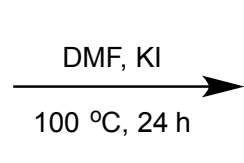<smiles>O=C1c2cc3ccccc3cc2C(=O)c2cc3c(Br)cccc3cc21</smiles>

1. $n$-BuLi, TIPS-acetylene THF, $-78{ }^{\circ} \mathrm{C}, 1 \mathrm{~h}$

2. 3, rt, $12 \mathrm{~h}$ followed by Satd. $\mathrm{SnCl}_{2}$ in $10 \% \mathrm{HCl}, 1 \mathrm{~h}$
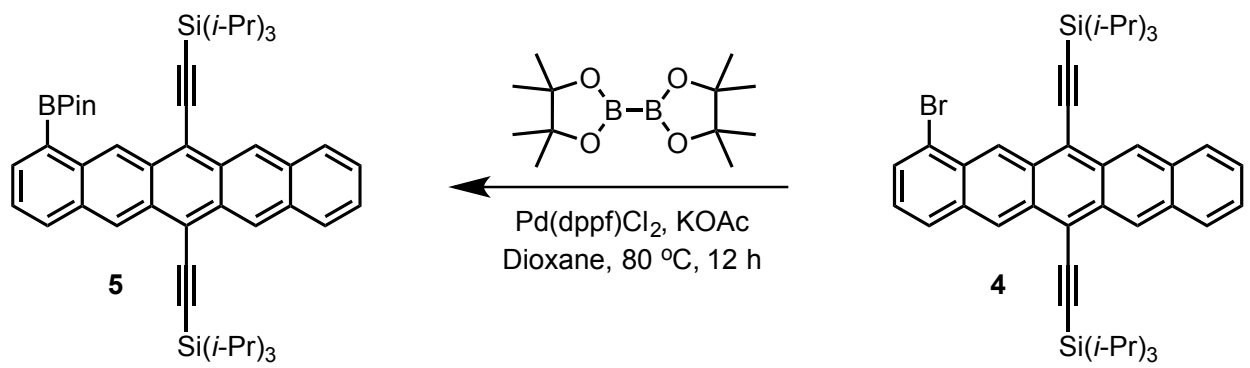

\section{Synthesis of Bipentacenes:}
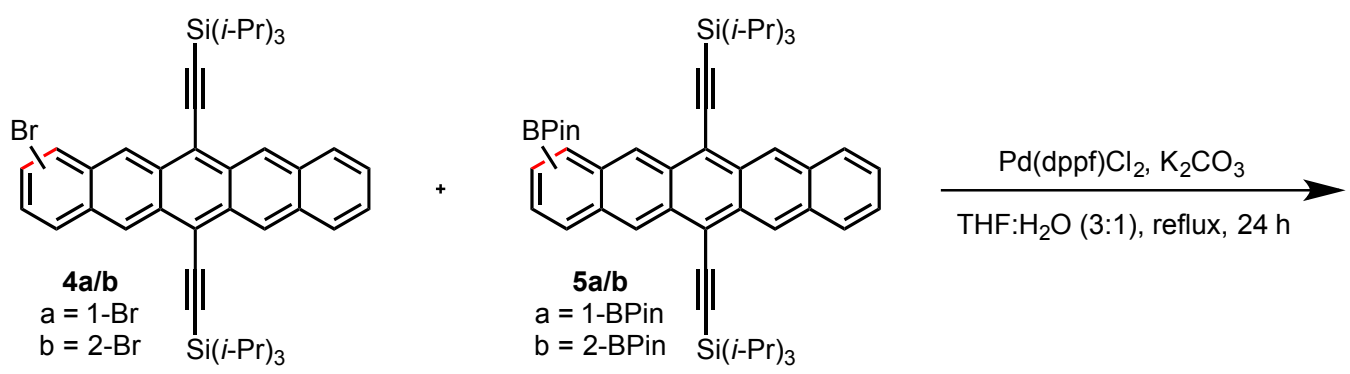

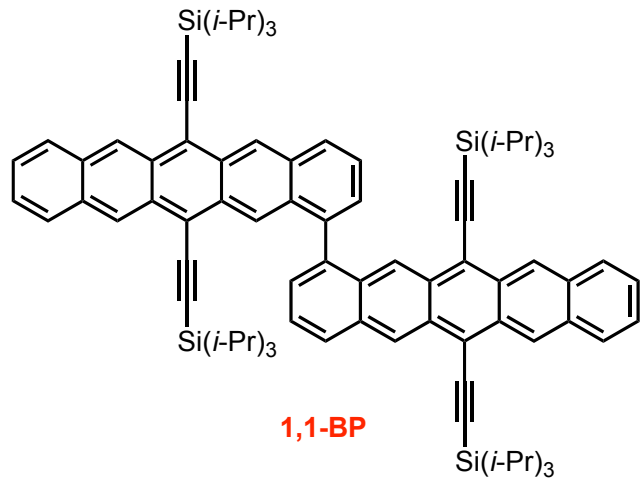

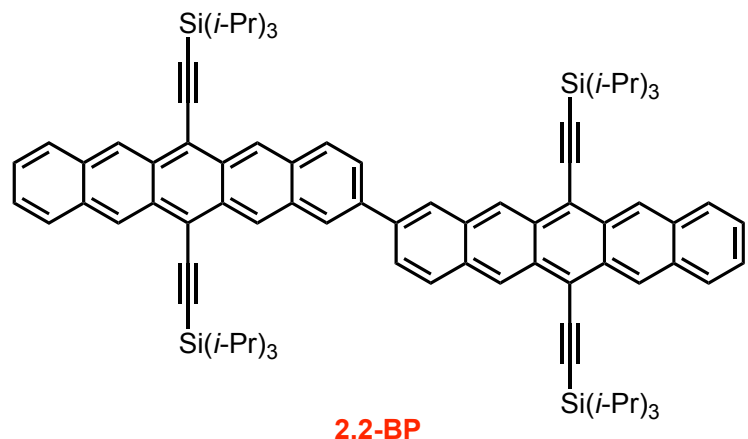

2,2-BP 


\section{General protocol for the synthesis of trans-ethylene-2,2-bipentacene}

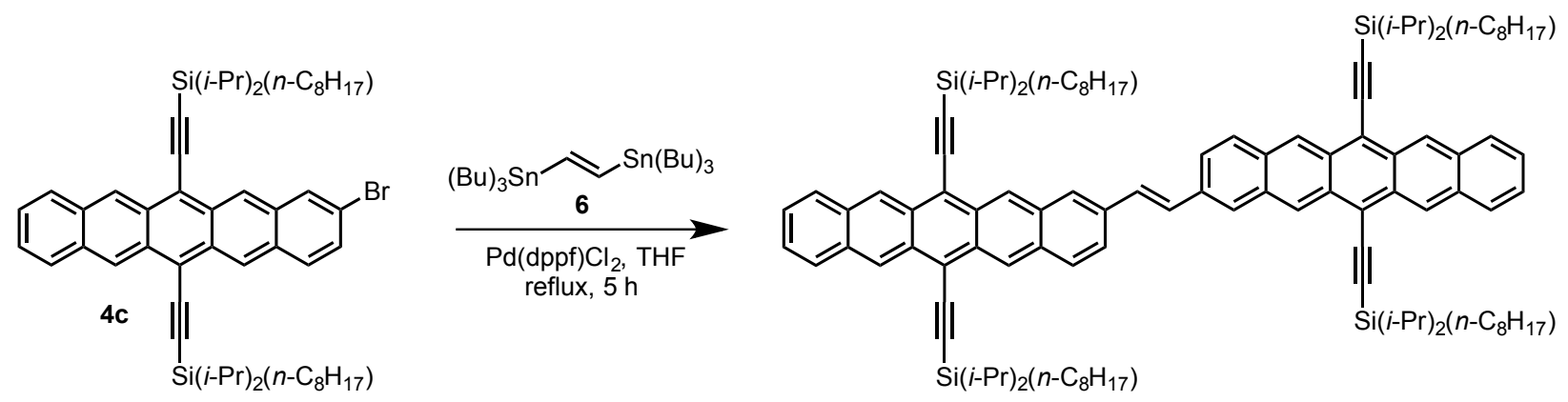

D. General procedure for the synthesis of BPin derivatives of pentacenes

1. Molecule 3

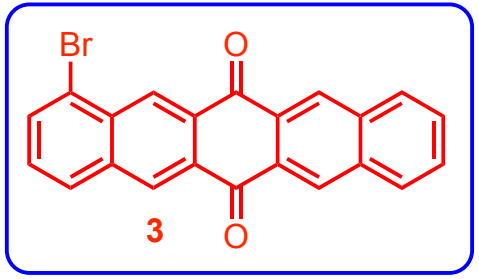

A mixture of 1,2-bis(dibromomethyl)benzene derivative 1 (1.1 equiv) and anthracene-1,4dione (1.0 equiv.) and $\mathrm{KI}$ (4.0 equiv.) in dry DMF was heated to $100^{\circ} \mathrm{C}$ and maintained for $24 \mathrm{~h}$. After the reaction, the mixture was cooled to room temperature and diluted with methanol. The slurry was filtered and the solid was washed with water and methanol. The resulting 6,13-pentacenequinone was collected and dried. The crude product was directly used for next step without further purification.

Due to limited solubility of the 6,13-pentacenequinones no characterization was carried out. 


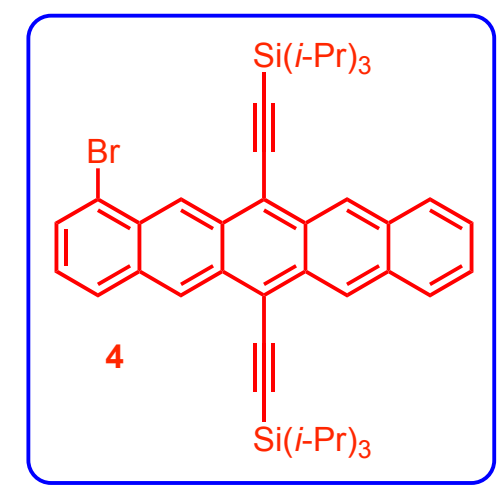

2. Molecule 4

To a solution of triisopropylsilylacetylene (3.5 equiv.) in dry and degassed THF (25 mL) in $200 \mathrm{~mL}$ Schlenk flask at $-78{ }^{\circ} \mathrm{C}$ added $n$-butyl lithium (3.4 equiv., $2.5 \mathrm{M}$ in hexanes). This solution was allowed to stir at $-78{ }^{\circ} \mathrm{C}$ for $1 \mathrm{~h}$ followed by the addition of $\mathbf{3}$ ( $4.0 \mathrm{~g}, 1.0$ equiv.) under positive argon flow. The solution was allowed to warm to rt and stirred overnight (16 h) or until solid pentacenoquinone was no longer observed. To this clear, deep yellow solution was added of a saturated solution of tin (II) chloride dihydrate in $10 \%$ aqueous $\mathrm{HCl}$ solution $(50 \mathrm{~mL})$ during which the solution turned deep blue. The resulting mixture was stirred at rt for $1 \mathrm{~h}$ under dark and filtered over a pad of silica. The solid was washed with DCM and the combined organic layer was washed with water $(2 \times 200 \mathrm{~mL})$, dried over anhyd. $\mathrm{Na}_{2} \mathrm{SO}_{4}$, filtered and the solvent was removed under reduced pressure to get the crude product. The crude was purified by silica chromatography using hexanes as an eluent to obtain bromopentacene derivative 4 as a deep blue solid in 16\% yield.

${ }^{1} \mathrm{H}-\mathrm{NMR}\left(500 \mathrm{MHz}, \mathrm{CDCl}_{3}, \delta \mathrm{ppm}\right): 9.72(\mathrm{~s}, 1 \mathrm{H}), 9.41-9.33(\mathrm{~m}, 3 \mathrm{H}), 8.04-7.96(\mathrm{~m}, 3 \mathrm{H})$, 7.77-7.75 $(\mathrm{m}, 1 \mathrm{H}), 7.48-7.45(2 \mathrm{H}), 7.29-7.25(\mathrm{~m}, 1 \mathrm{H})$ and 1.48-1.38 $(\mathrm{m}, 42 \mathrm{H})$.

${ }^{13} \mathrm{C}-\mathrm{NMR}\left(125 \mathrm{MHz}, \mathrm{CDCl}_{3}, \delta \mathrm{ppm}\right): 132.8,132.5,132.4,130.94,130.9,130.8,132.75$, $130.72,129.8,128.9,128.7,128.66,127.3,126.9,126.6,126.3,126.3,126.2,125.8,123.4$, 119.3, 118.3, 108.0, 107.5, 104.5, 104.3, 19.1, 19.0 and 11.7.

MS (ASAP): Calculated: 717.2947; Observed: 717.2939. 


\section{Molecule 5}

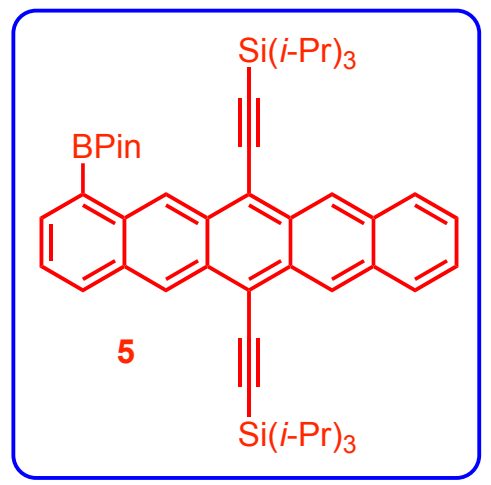

To a dry round bottomed flask was added 4 (4.0 g, $5.57 \mathrm{mmol}), \mathrm{Pd}(\mathrm{dppf}) \mathrm{Cl}_{2} \cdot \mathrm{DCM}(203$ $\mathrm{mg}, 0.25 \mathrm{mmol})$, KOAc (1.91 g, $19.5 \mathrm{mmol})$, and bis(pinacolato)diboron (2.82g, $11.1 \mathrm{mmol})$. Sequential vacuum and argon were used to degas the mixture followed by the addition of degassed 1, 4 dioxane $(70 \mathrm{~mL})$. The mixture was heated to $80{ }^{\circ} \mathrm{C}$ and maintained for $12 \mathrm{~h}$ in the dark. After the reaction, the mixture was cooled to rt and the solvent was removed under reduced pressure. The crude was partitioned between DCM (250 mL) and water $(200 \mathrm{~mL})$. The organic layer was separated, washed with water $(2 \times 200 \mathrm{~mL})$, dried over anhyd. $\mathrm{Na}_{2} \mathrm{SO}_{4}$, filtered and the solvent was removed under reduced pressure to get the crude product. The crude was purified by silica chromatography using mixtures of hexanes/DCM as an eluent to obtain BPin pentacene derivative $\mathbf{5}$ as a deep blue solid in $80 \%$ yield.

${ }^{1} \mathrm{H}-\mathrm{NMR}\left(400 \mathrm{MHz}, \mathrm{CDCl}_{3}, \delta \mathrm{ppm}\right): 9.93$ (s, 1H), 9.52 (s, 1H), 9.37 (s, 2H), 8.12-8.08 (m, 2H), 8.04-8.00 (m, 2H), 7.47-7.44 (m, 3H), $1.54(\mathrm{~s}, 12 \mathrm{H})$ and 1.44-1.40 (m, 42H).

${ }^{13} \mathrm{C}-\mathrm{NMR}\left(100 \mathrm{MHz}, \mathrm{CDCl}_{3}, \delta \mathrm{ppm}\right):$ 137.4, 134.5, 132.6, 132.4, 132.3, 132.2, 131.6, 130.7, 130.6, 130.4, 128.6, 127.3, 126.8, 126.7, 126.2, 125.96, 125.1, 118.9, 118.3, 107.6, 107.0, 105.5, $104.9,83.9,24.9,19.2,19.0,12.0$ and 11.7 .

MS (ASAP): Calculated: 765.4694; Observed: 765.4688 


\section{E. Synthesis of bipentacenes:}

To a $20 \mathrm{~mL}$ sealed tube was added $\mathbf{4 a} / \mathbf{b}$ (72 $\mathrm{mg}, 0.1 \mathrm{mmol}), \mathbf{5 a} / \mathbf{b}(76 \mathrm{mg}, 0.1 \mathrm{mmol})$, $\mathrm{Pd}(\mathrm{dppf}) \mathrm{Cl}_{2} \cdot \mathrm{DCM}(4 \mathrm{mg}, 0.005 \mathrm{mmol})$, and $\mathrm{K}_{2} \mathrm{CO}_{3}(240 \mathrm{mg}, 1.7 \mathrm{mmol})$. Sequential vacuum and argon were used to degas the mixture followed by the addition of degassed H2O (1 mL) and THF $(3 \mathrm{~mL})$. The resulting solution was heated to $70{ }^{\circ} \mathrm{C}$ and maintained for $24 \mathrm{~h}$ in dark. After the reaction, the solution was poured into a separatory funnel containing DCM $(30 \mathrm{~mL})$ and water $(30 \mathrm{~mL})$. The organic layer was separated, dried over anhyd. $\mathrm{Na}_{2} \mathrm{SO}_{4}$,

filtered and the solvent was removed under reduced pressure to get the crude product. The crude was purified by silica chromatography using mixtures of hexanes/DCM as an eluent to obtain bis-pentacenes as solid.

\section{1. $11 B P$}

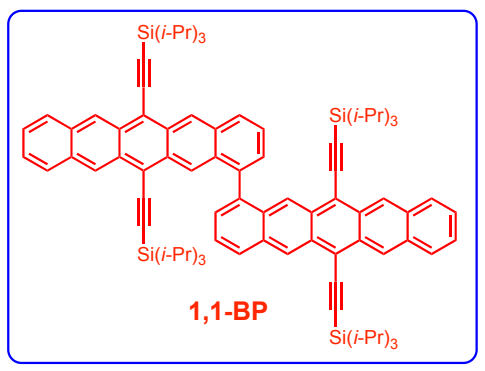

Yield: $84 \%$ (Pale blue solid)

${ }^{1} \mathrm{H}-\mathrm{NMR}\left(500 \mathrm{MHz}, \mathrm{CDCl}_{3}, \delta \mathrm{ppm}\right): 9.51$ (s, 1H), 9.31 (s, 1H), 9.15 (s, 1H), 9.07 (s, 1H), $8.15(\mathrm{~d}, J=8.5 \mathrm{~Hz}, 1 \mathrm{H}), 7.97(\mathrm{~d}, J=8.5 \mathrm{~Hz}, 1 \mathrm{H}), 7.85(\mathrm{~d}, J=8.5 \mathrm{~Hz}, 1 \mathrm{H}), 7.61-7.58$ $(\mathrm{m}, 1 \mathrm{H}), 7.52-7.51(\mathrm{~m}, 1 \mathrm{H}), 7.41-7.34(\mathrm{~m}, 2 \mathrm{H}), 1.496-1.44(\mathrm{~m}, 21 \mathrm{H}), 0.87-0.86(\mathrm{~m}, 9 \mathrm{H})$ and 0.76-0.68 (m, 12H).

${ }^{13} \mathrm{C}-\mathrm{NMR}\left(125 \mathrm{MHz}, \mathrm{CDCl}_{3}, \delta \mathrm{ppm}\right): 139.3,132.7,132.3,132.2,132.1,130.8,130.6$, 129.1, 128.7, 128.6, 128.2, 126.9, 126.5, 126.1, 125.9, 125.9, 125.8, 125.7, 118.9, 117.9, 107.4, 106.8, 104.9, 103.8, 19.1, 18.6, 18.5, 11.8 and 11.2.

MS (ASAP): Calculated: 1275.7450; Observed: 1275.7440 


\section{2. $22 e B P$}

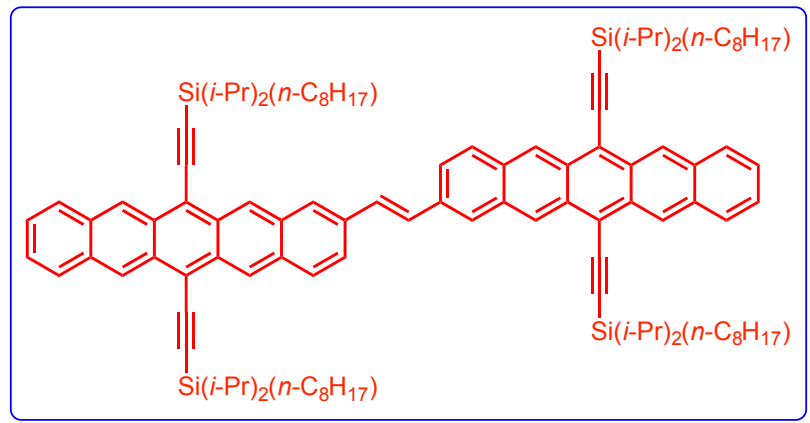

To a $20 \mathrm{~mL}$ sealed tube was added $4 \mathbf{c}(240 \mathrm{mg}, 0.28 \mathrm{mmol}), \operatorname{Pd}(\mathrm{dppf}) \mathrm{Cl}_{2} \cdot \mathrm{DCM}(12 \mathrm{mg}$, $0.015 \mathrm{mmol}$ ), and trans-1,2-bis(tri-n-butylstannyl)ethylene 6 (66 mg, $0.11 \mathrm{mmol})$. Sequential vacuum and argon were used to degas the mixture followed by the addition of degassed THF $(3 \mathrm{~mL})$. The mixture was heated to reflux and maintained for $5 \mathrm{~h}$ in the dark. After the reaction, the mixture was cooled to $\mathrm{rt}$ and the solvent was removed under reduced pressure. The crude reaction mixture was purified by silica chromatography using mixtures of hexanes/DCM as an eluent to obtain product as a deep blue solid with $80 \%$ yield.

${ }^{1} \mathrm{H}-\mathrm{NMR}\left(500 \mathrm{MHz}, \mathrm{CDCl}_{3}, \delta \mathrm{ppm}\right): 9.32-9.23(\mathrm{~m}, 8 \mathrm{H}), 8.02-7.97(\mathrm{~m}, 8 \mathrm{H}), 7.86-7.84$ $(\mathrm{m}, 2 \mathrm{H}), 7.50(\mathrm{~s}, 2 \mathrm{H}), 7.49-7.42(\mathrm{~m}, 4 \mathrm{H}), 1.85-1.77(\mathrm{~m}, 8 \mathrm{H}), 1.58-1.54\left(8 \mathrm{H}\right.$, overlap $\left.\mathrm{H}_{2} \mathrm{O}\right)$, $1.42-1.34(\mathrm{~m}, 70 \mathrm{H}), 1.29-1.27(\mathrm{~m}, 16 \mathrm{H}), 1.03-0.98(\mathrm{~m}, 9 \mathrm{H})$ and $0.89-0.81(\mathrm{~m}, 13 \mathrm{H})$

${ }^{13} \mathrm{C}-\mathrm{NMR}\left(125 \mathrm{MHz}, \mathrm{CDCl}_{3}, \delta \mathrm{ppm}\right): 134.6,132.4,132.3,132.2,131.9,131.0,130.8$, $130.7,130.5,129.1,128.6,128.2,126.4,126.3,126.2$, 126.0, 125.99, 125.91, 123.1, 118.4, 118.2, 107.4, 107.3, 104.6, 104.5, 34.1, 34.07, 32.0, 29.6, 29.5, 29.44, 29.41, 25.0, 24.99, 22.7, $18.8,18.77,18.72,18.5,18.49,14.1,14.08,12.3,12.2,10.6$ and 10.5 .

MS (ESI): Calculated: 1582.0712; Observed: 1582.0714 


\section{CO-ORDINATES FOR ELECTRONIC STRUCTURE CALCULATIONS}

All coordinates are given in Ångströms in GAMESS notation.

\section{A. TIPS-pentacene}

Optimized in the $D_{2 h}$ point group.

\begin{tabular}{|c|c|c|c|c|}
\hline ATOM & CHARGE & $\mathrm{X}$ & Y & $\mathrm{Z}$ \\
\hline & 6.0 & 0.0000000000 & 4.0308083610 & 0.0000000000 \\
\hline & 6.0 & 0.0000000000 & -4.0308083610 & 0.0000000000 \\
\hline & 6.0 & 0.0000000000 & 2.8480468252 & 0.0000000000 \\
\hline & 6.0 & 0.0000000000 & -2.8480468252 & 0.0000000000 \\
\hline & 6.0 & 0.0000000000 & 1.4087548890 & 0.0000000000 \\
\hline & 6.0 & -0.0000000000 & -1.4087548890 & 0.0000000000 \\
\hline & 6.0 & -1.2241740825 & 0.7138331268 & 0.0000000000 \\
\hline & 6.0 & 1.2241740825 & 0.7138331268 & 0.0000000000 \\
\hline & 6.0 & -1.2241740825 & -0.7138331268 & 0.0000000000 \\
\hline & 6.0 & 1.2241740825 & -0.7138331268 & 0.0000000000 \\
\hline & 6.0 & -2.4645223735 & 1.3937263190 & 0.0000000000 \\
\hline & 6.0 & 2.4645223735 & 1.3937263190 & 0.0000000000 \\
\hline & 6.0 & -2.4645223735 & -1.3937263190 & 0.0000000000 \\
\hline & 6.0 & 2.4645223735 & -1.3937263190 & 0.0000000000 \\
\hline & 6.0 & -3.6641994773 & 0.7090627815 & 0.0000000000 \\
\hline & 6.0 & 3.6641994773 & 0.7090627815 & 0.0000000000 \\
\hline & 6.0 & -3.6641994773 & -0.7090627815 & 0.0000000000 \\
\hline & 6.0 & 3.6641994773 & -0.7090627815 & 0.0000000000 \\
\hline & 6.0 & -6.0812759032 & 0.7175412661 & 0.0000000000 \\
\hline & 6.0 & 6.0812759032 & 0.7175412661 & 0.0000000000 \\
\hline & 6.0 & -6.0812759032 & -0.7175412661 & 0.0000000000 \\
\hline
\end{tabular}




$\begin{array}{lrrrr}\mathrm{C} & 6.0 & 6.0812759032 & -0.7175412661 & 0.00000000000 \\ \mathrm{C} & 6.0 & -4.9258450649 & 1.3986591718 & 0.00000000000 \\ \mathrm{C} & 6.0 & 4.9258450649 & 1.3986591718 & 0.0000000000 \\ \mathrm{C} & 6.0 & -4.9258450649 & -1.3986591718 & 0.00000000000 \\ \mathrm{C} & 6.0 & 4.9258450649 & -1.3986591718 & 0.0000000000 \\ \mathrm{H} & 1.0 & 0.0000000000 & 5.0839495280 & 0.0000000000 \\ \mathrm{H} & 1.0 & 0.0000000000 & -5.0839495280 & 0.0000000000 \\ \mathrm{H} & 1.0 & -2.4695833345 & 2.4631338945 & 0.0000000000 \\ \mathrm{H} & 1.0 & 2.4695833345 & 2.4631338945 & 0.00000000000 \\ \mathrm{H} & 1.0 & -2.4695833345 & -2.4631338945 & 0.00000000000 \\ \mathrm{H} & 1.0 & 2.4695833345 & -2.4631338945 & 0.00000000000 \\ \mathrm{H} & 1.0 & -4.9230541382 & 2.4715419620 & 0.0000000000 \\ \mathrm{H} & 1.0 & 4.9230541382 & 2.4715419620 & 0.0000000000 \\ \mathrm{H} & 1.0 & -4.9230541382 & -2.4715419620 & 0.0000000000 \\ \mathrm{H} & 1.0 & 4.9230541382 & -2.4715419620 & 0.0000000000 \\ \mathrm{H} & 1.0 & 7.0179156581 & -1.2400824719 & 0.00000000000 \\ \mathrm{H} & 1.0 & -7.0179156581 & -1.2400824719 & 0.0000000000 \\ \mathrm{H} & 1.0 & 7.0179156581 & 1.2400824719 & 0.00000000000 \\ \mathrm{H} & 1.0 & -7.0179156581 & 1.2400824719 & 0.00000000000\end{array}$

\section{B. 11BP}

Optimized in the $C_{2}$ point group. A number of low-energy structures were found with slightly different dihedral angles $\left(80^{\circ}-100^{\circ}\right)$ between the two pentacenes, but which did not significantly alter the calculated spectra. We chose a structure with a dihedral of $81^{\circ}$ which was supported by low-level calculations on 11BP with the TIPS groups.
C
$6.0 \quad-5.0168112146 \quad 1.6786790667$
0.0464831369
$\mathrm{C}$
$\begin{array}{llll}6.0 & 5.0168112146 & -1.6786790667 & 0.0464831369\end{array}$
C
$\begin{array}{lllll}6.0 & -3.7770489120 & -0.8995243633 & -0.0234142169\end{array}$ 


\begin{tabular}{|c|c|c|c|c|}
\hline $\mathrm{C}$ & 6.0 & 3.7770489120 & 0.8995243633 & -0.0234142169 \\
\hline $\mathrm{C}$ & 6.0 & -5.6341113352 & 2.9610319681 & 0.0836332259 \\
\hline $\mathrm{C}$ & 6.0 & 5.6341113352 & -2.9610319681 & 0.0836332259 \\
\hline $\mathrm{C}$ & 6.0 & -3.1608849614 & -2.1824592852 & -0.0580493116 \\
\hline $\mathrm{C}$ & 6.0 & 3.1608849614 & 2.1824592852 & -0.0580493116 \\
\hline $\mathrm{C}$ & 6.0 & -6.1618397466 & 4.0524937895 & 0.1147753046 \\
\hline $\mathrm{C}$ & 6.0 & 6.1618397466 & -4.0524937895 & 0.1147753046 \\
\hline $\mathrm{C}$ & 6.0 & -2.6362554178 & -3.2751541152 & -0.0859442496 \\
\hline $\mathrm{C}$ & 6.0 & 2.6362554178 & 3.2751541152 & -0.0859442496 \\
\hline $\mathrm{H}$ & 1.0 & -6.6270642889 & 5.0117618599 & 0.1402879113 \\
\hline $\mathrm{H}$ & 1.0 & 6.6270642889 & -5.0117618599 & 0.1402879113 \\
\hline $\mathrm{H}$ & 1.0 & -2.1654838949 & -4.2317995341 & -0.1083126340 \\
\hline $\mathrm{H}$ & 1.0 & 2.1654838949 & 4.2317995341 & -0.1083126340 \\
\hline $\mathrm{C}$ & 6.0 & -5.5622687669 & 0.6571259705 & -0.7810752597 \\
\hline $\mathrm{C}$ & 6.0 & 5.5622687669 & -0.6571259705 & -0.7810752597 \\
\hline $\mathrm{C}$ & 6.0 & -3.8603762837 & 1.4291449469 & 0.8376328753 \\
\hline $\mathrm{C}$ & 6.0 & 3.8603762837 & -1.4291449469 & 0.8376328753 \\
\hline C & 6.0 & -4.9366736721 & -0.6530391474 & -0.8115082957 \\
\hline $\mathrm{C}$ & 6.0 & 4.9366736721 & 0.6530391474 & -0.8115082957 \\
\hline $\mathrm{C}$ & 6.0 & -3.2276382667 & 0.1245364571 & 0.7985422638 \\
\hline $\mathrm{C}$ & 6.0 & 3.2276382667 & -0.1245364571 & 0.7985422638 \\
\hline $\mathrm{C}$ & 6.0 & -6.7048631222 & 0.8805966134 & -1.5805194842 \\
\hline $\mathrm{C}$ & 6.0 & 6.7048631222 & -0.8805966134 & -1.5805194842 \\
\hline $\mathrm{C}$ & 6.0 & -3.2998609203 & 2.4214212570 & 1.6698352866 \\
\hline C & 6.0 & 3.2998609203 & -2.4214212570 & 1.6698352866 \\
\hline $\mathrm{C}$ & 6.0 & -5.5017716946 & -1.6548483714 & -1.6313085290 \\
\hline C & 6.0 & 5.5017716946 & 1.6548483714 & -1.6313085290 \\
\hline $\mathrm{C}$ & 6.0 & -2.0748711290 & -0.0989087129 & 1.5847195629 \\
\hline $\mathrm{C}$ & 6.0 & 2.0748711290 & 0.0989087129 & 1.5847195629 \\
\hline $\mathrm{H}$ & 1.0 & -7.1682027183 & 1.8623796989 & -1.5602718633 \\
\hline
\end{tabular}




\begin{tabular}{|c|c|c|c|c|}
\hline $\mathrm{H}$ & 1.0 & 7.1682027183 & -1.8623796989 & -1.5602718633 \\
\hline $\mathrm{H}$ & 1.0 & -3.7724288545 & 3.3985212045 & 1.7025429323 \\
\hline $\mathrm{H}$ & 1.0 & 3.7724288545 & -3.3985212045 & 1.7025429323 \\
\hline $\mathrm{H}$ & 1.0 & -5.0342209852 & -2.6346700386 & -1.6485433777 \\
\hline $\mathrm{H}$ & 1.0 & 5.0342209852 & 2.6346700386 & -1.6485433777 \\
\hline $\mathrm{H}$ & 1.0 & -1.6088469264 & -1.0763983682 & 1.5451099309 \\
\hline $\mathrm{H}$ & 1.0 & 1.6088469264 & 1.0763983682 & 1.5451099309 \\
\hline $\mathrm{C}$ & 6.0 & -7.2493988858 & -0.1126690066 & -2.3876427575 \\
\hline $\mathrm{C}$ & 6.0 & 7.2493988858 & 0.1126690066 & -2.3876427575 \\
\hline $\mathrm{C}$ & 6.0 & -2.1669125893 & 2.1903869510 & 2.4432341397 \\
\hline $\mathrm{C}$ & 6.0 & 2.1669125893 & -2.1903869510 & 2.4432341397 \\
\hline $\mathrm{C}$ & 6.0 & -6.6303294657 & -1.4242279454 & -2.4106306834 \\
\hline $\mathrm{C}$ & 6.0 & 6.6303294657 & 1.4242279454 & -2.4106306834 \\
\hline $\mathrm{C}$ & 6.0 & -1.5280677528 & 0.8865209381 & 2.4013913396 \\
\hline $\mathrm{C}$ & 6.0 & 1.5280677528 & -0.8865209381 & 2.4013913396 \\
\hline $\mathrm{C}$ & 6.0 & -8.4084149891 & 0.1132809950 & -3.2031695924 \\
\hline $\mathrm{C}$ & 6.0 & 8.4084149891 & -0.1132809950 & -3.2031695924 \\
\hline $\mathrm{C}$ & 6.0 & -1.6120311759 & 3.2120194516 & 3.2823688117 \\
\hline $\mathrm{C}$ & 6.0 & 1.6120311759 & -3.2120194516 & 3.2823688117 \\
\hline $\mathrm{C}$ & 6.0 & -7.2071942329 & -2.4409706094 & -3.2433249054 \\
\hline $\mathrm{C}$ & 6.0 & 7.2071942329 & 2.4409706094 & -3.2433249054 \\
\hline $\mathrm{C}$ & 6.0 & -0.3437935893 & 0.6649544380 & 3.2076966897 \\
\hline $\mathrm{C}$ & 6.0 & 0.3437935893 & -0.6649544380 & 3.2076966897 \\
\hline $\mathrm{H}$ & 1.0 & -8.8683908544 & 1.0986165748 & -3.1862532429 \\
\hline $\mathrm{H}$ & 1.0 & 8.8683908544 & -1.0986165748 & -3.1862532429 \\
\hline $\mathrm{H}$ & 1.0 & -2.1010646062 & 4.1830056111 & 3.3088108062 \\
\hline $\mathrm{H}$ & 1.0 & 2.1010646062 & -4.1830056111 & 3.3088108062 \\
\hline $\mathrm{H}$ & 1.0 & -6.7426838926 & -3.4242233517 & -3.2556394540 \\
\hline $\mathrm{H}$ & 1.0 & 6.7426838926 & 3.4242233517 & -3.2556394540 \\
\hline C & 6.0 & -8.9228223218 & -0.8846949932 & -3.9831660604 \\
\hline
\end{tabular}




$\begin{array}{lrrrr}\mathrm{C} & 6.0 & 8.9228223218 & 0.8846949932 & -3.9831660604 \\ \mathrm{C} & 6.0 & -0.4946723195 & 2.9676969390 & 4.0285519904 \\ \mathrm{C} & 6.0 & 0.4946723195 & -2.9676969390 & 4.0285519904 \\ \mathrm{C} & 6.0 & -8.3140535576 & -2.1814329492 & -4.0025104129 \\ \mathrm{C} & 6.0 & 8.3140535576 & 2.1814329492 & -4.0025104129 \\ \mathrm{C} & 6.0 & 0.1409618000 & 1.6875984915 & 3.9854886973 \\ \mathrm{C} & 6.0 & -0.1409618000 & -1.6875984915 & 3.9854886973 \\ \mathrm{H} & 1.0 & -9.8016592260 & -0.7011761381 & -4.5958530875 \\ \mathrm{H} & 1.0 & 9.8016592260 & 0.7011761381 & -4.5958530875 \\ \mathrm{H} & 1.0 & -0.0768503612 & 3.7433357089 & 4.6650280256 \\ \mathrm{H} & 1.0 & 0.0768503612 & -3.7433357089 & 4.6650280256 \\ \mathrm{H} & 1.0 & -8.7419485671 & -2.9596339230 & -4.6292654463 \\ \mathrm{H} & 1.0 & 8.7419485671 & 2.9596339230 & -4.6292654463 \\ \mathrm{H} & 1.0 & 1.0306306985 & 1.5196266204 & 4.5868899340 \\ \mathrm{H} & 1.0 & -1.0306306985 & -1.5196266204 & 4.5868899340\end{array}$

\section{22BP}

Structure taken from Ref. [1].

\section{22eBP}

Optimized in the $C_{2 h}$ point group.

$\begin{array}{lrrrr}\text { C } & 6.0 & -7.92668247 & -1.26516759 & 0.00000000 \\ \text { C } & 6.0 & 7.92667723 & 1.26516676 & 0.00000000 \\ \text { C } & 6.0 & -8.31315231 & 1.56966603 & 0.00000000 \\ \text { C } & 6.0 & 8.31314659 & -1.56966686 & 0.00000000 \\ \text { C } & 6.0 & -7.73448944 & -2.67577052 & 0.00000000 \\ \text { C } & 6.0 & 7.73448372 & 2.67576981 & 0.00000000 \\ \text { C } & 6.0 & -8.50554085 & 2.98072267 & 0.00000000\end{array}$




\begin{tabular}{|c|c|c|c|c|}
\hline $\mathrm{C}$ & 6.0 & 8.50553513 & -2.98072338 & 0.00000000 \\
\hline $\mathrm{C}$ & 6.0 & -7.57011127 & -3.87732530 & 0.00000000 \\
\hline $\mathrm{C}$ & 6.0 & 7.57005262 & 3.87731624 & 0.00000000 \\
\hline $\mathrm{C}$ & 6.0 & -8.67098522 & 4.18200779 & 0.00000000 \\
\hline $\mathrm{C}$ & 6.0 & 8.67100811 & -4.18200445 & 0.00000000 \\
\hline $\mathrm{H}$ & 1.0 & -7.42592239 & -4.93404913 & 0.00000000 \\
\hline $\mathrm{H}$ & 1.0 & 7.42644835 & 4.93412924 & 0.00000000 \\
\hline $\mathrm{H}$ & 1.0 & -8.81589413 & 5.23868799 & 0.00000000 \\
\hline $\mathrm{H}$ & 1.0 & 8.81561184 & -5.23873091 & 0.00000000 \\
\hline $\mathrm{C}$ & 6.0 & -9.24816132 & -0.73380011 & 0.00000000 \\
\hline $\mathrm{C}$ & 6.0 & 9.24815559 & 0.73379898 & 0.00000000 \\
\hline $\mathrm{C}$ & 6.0 & -6.79677439 & -0.40012172 & 0.00000000 \\
\hline $\mathrm{C}$ & 6.0 & 6.79676867 & 0.40012118 & 0.00000000 \\
\hline $\mathrm{C}$ & 6.0 & -9.44531250 & 0.70449984 & 0.00000000 \\
\hline $\mathrm{C}$ & 6.0 & 9.44530773 & -0.70450091 & 0.00000000 \\
\hline $\mathrm{C}$ & 6.0 & -6.99528408 & 1.03862977 & 0.00000000 \\
\hline $\mathrm{C}$ & 6.0 & 6.99527884 & -1.03863037 & 0.00000000 \\
\hline $\mathrm{C}$ & 6.0 & -10.38371086 & -1.57286096 & 0.00000000 \\
\hline $\mathrm{C}$ & 6.0 & 10.38370514 & 1.57286048 & 0.00000000 \\
\hline $\mathrm{C}$ & 6.0 & -5.47686577 & -0.90274304 & 0.00000000 \\
\hline $\mathrm{C}$ & 6.0 & 5.47686005 & 0.90274173 & 0.00000000 \\
\hline $\mathrm{C}$ & 6.0 & -10.76369190 & 1.20770228 & 0.00000000 \\
\hline $\mathrm{C}$ & 6.0 & 10.76368713 & -1.20770264 & 0.00000000 \\
\hline $\mathrm{C}$ & 6.0 & -5.85674047 & 1.87935269 & 0.00000000 \\
\hline $\mathrm{C}$ & 6.0 & 5.85673523 & -1.87935376 & 0.00000000 \\
\hline $\mathrm{H}$ & 1.0 & -10.23241329 & -2.64811635 & 0.00000000 \\
\hline $\mathrm{H}$ & 1.0 & 10.23240566 & 2.64811540 & 0.00000000 \\
\hline $\mathrm{H}$ & 1.0 & -5.33027649 & -1.97862923 & 0.00000000 \\
\hline $\mathrm{H}$ & 1.0 & 5.33026886 & 1.97862792 & 0.00000000 \\
\hline $\mathrm{H}$ & 1.0 & -10.90994263 & 2.28368711 & 0.00000000 \\
\hline
\end{tabular}




\begin{tabular}{|c|c|c|c|c|}
\hline $\mathrm{H}$ & 1.0 & 10.90993786 & -2.28368759 & 0.00000000 \\
\hline $\mathrm{H}$ & 1.0 & -6.00853205 & 2.95449662 & 0.00000000 \\
\hline $\mathrm{H}$ & 1.0 & 6.00852776 & -2.95449758 & 0.00000000 \\
\hline $\mathrm{C}$ & 6.0 & -11.67914391 & -1.06567049 & 0.00000000 \\
\hline $\mathrm{C}$ & 6.0 & 11.67913818 & 1.06567049 & 0.00000000 \\
\hline $\mathrm{C}$ & 6.0 & -4.36550093 & -0.06438924 & 0.00000000 \\
\hline C & 6.0 & 4.36549520 & 0.06438758 & 0.00000000 \\
\hline C & 6.0 & -11.87567043 & 0.37100893 & 0.00000000 \\
\hline C & 6.0 & 11.87566471 & -0.37100887 & 0.00000000 \\
\hline $\mathrm{C}$ & 6.0 & -4.56643677 & 1.37154436 & 0.00000000 \\
\hline $\mathrm{C}$ & 6.0 & 4.56643152 & -1.37154591 & 0.00000000 \\
\hline $\mathrm{C}$ & 6.0 & -12.83485413 & -1.91612911 & 0.00000000 \\
\hline $\mathrm{C}$ & 6.0 & 12.83484745 & 1.91612971 & 0.00000000 \\
\hline $\mathrm{C}$ & 6.0 & -3.02918434 & -0.56868756 & 0.00000000 \\
\hline $\mathrm{C}$ & 6.0 & 3.02917838 & 0.56868529 & 0.00000000 \\
\hline C & 6.0 & -13.21658802 & 0.88056803 & 0.00000000 \\
\hline C & 6.0 & 13.21658325 & -0.88056731 & 0.00000000 \\
\hline $\mathrm{C}$ & 6.0 & -3.40303636 & 2.21517420 & 0.00000000 \\
\hline $\mathrm{C}$ & 6.0 & 3.40303159 & -2.21517611 & 0.00000000 \\
\hline $\mathrm{H}$ & 1.0 & -12.68388176 & -2.99316859 & 0.00000000 \\
\hline $\mathrm{H}$ & 1.0 & 12.68387508 & 2.99316907 & 0.00000000 \\
\hline $\mathrm{H}$ & 1.0 & -2.88808918 & -1.64788556 & 0.00000000 \\
\hline $\mathrm{H}$ & 1.0 & 2.88808250 & 1.64788330 & 0.00000000 \\
\hline $\mathrm{H}$ & 1.0 & -13.36110401 & 1.95852780 & 0.00000000 \\
\hline $\mathrm{H}$ & 1.0 & 13.36109924 & -1.95852697 & 0.00000000 \\
\hline $\mathrm{H}$ & 1.0 & -3.54865170 & 3.29301834 & 0.00000000 \\
\hline $\mathrm{H}$ & 1.0 & 3.54864740 & -3.29302025 & 0.00000000 \\
\hline $\mathrm{C}$ & 6.0 & -14.09543991 & -1.38647938 & 0.00000000 \\
\hline $\mathrm{C}$ & 6.0 & 14.09543324 & 1.38648045 & 0.00000000 \\
\hline & 6.0 & -1.92513561 & 0.26085106 & 0.00000000 \\
\hline
\end{tabular}




$\begin{array}{lrrrr}\text { C } & 6.0 & 1.92513001 & -0.26085377 & 0.00000000 \\ \text { C } & 6.0 & -14.28925800 & 0.03266053 & 0.00000000 \\ \text { C } & 6.0 & 14.28925323 & -0.03265931 & 0.00000000 \\ \text { C } & 6.0 & -2.14609814 & 1.69066441 & 0.00000000 \\ \text { C } & 6.0 & 2.14609313 & -1.69066703 & 0.00000000 \\ \text { H } & 1.0 & -14.96322536 & -2.04089022 & 0.00000000 \\ \mathrm{H} & 1.0 & 14.96321869 & 2.04089165 & 0.00000000 \\ \mathrm{H} & 1.0 & -15.30041599 & 0.43142483 & 0.00000000 \\ \mathrm{H} & 1.0 & 15.30041027 & -0.43142313 & 0.00000000 \\ \mathrm{H} & 1.0 & -1.29143643 & 2.35977221 & 0.00000000 \\ \mathrm{H} & 1.0 & 1.29143167 & -2.35977530 & 0.00000000 \\ \mathrm{C} & 6.0 & -0.59145021 & -0.32948795 & 0.00000000 \\ \mathrm{C} & 6.0 & 0.59144425 & 0.32948473 & 0.00000000 \\ \mathrm{H} & 1.0 & -0.58765703 & -1.41819751 & 0.00000000 \\ \mathrm{H} & 1.0 & 0.58765107 & 1.41819429 & 0.00000000\end{array}$

\section{E. $66 y B P$}

This structure was optimized in the $D_{2 h}$ point group. Test calculations were performed on a twisted structure $\left(D_{2}\right.$ symmetry) and found it of very slightly higher energy.

$\begin{array}{lrrrr}\text { C } & 6.0 & 8.8126464405 & 0.0000000000 & 0.0000000000 \\ \text { C } & 6.0 & -8.8126464405 & 0.0000000000 & 0.0000000000 \\ \text { C } & 6.0 & 0.6754618538 & 0.0000000000 & 0.0000000000 \\ \text { C } & 6.0 & -0.6754618538 & 0.0000000000 & 0.0000000000 \\ C & 6.0 & 7.5997592132 & 0.0000000000 & 0.0000000000 \\ \text { C } & 6.0 & -7.5997592132 & 0.0000000000 & 0.0000000000 \\ \text { C } & 6.0 & 1.9039435613 & 0.0000000000 & 0.0000000000 \\ \text { C } & 6.0 & -1.9039435613 & 0.0000000000 & 0.0000000000 \\ \text { C } & 6.0 & 6.1769124108 & 0.0000000000 & 0.0000000000 \\ \text { C } & 6.0 & -6.1769124108 & 0.0000000000 & 0.0000000000\end{array}$




\begin{tabular}{|c|c|c|c|c|}
\hline $\mathrm{C}$ & 6.0 & 3.3101132040 & 0.0000000000 & 0.0000000000 \\
\hline $\mathrm{C}$ & 6.0 & -3.3101132040 & 0.0000000000 & 0.0000000000 \\
\hline $\mathrm{C}$ & 6.0 & 5.4725886874 & -1.2381061438 & 0.0000000000 \\
\hline $\mathrm{C}$ & 6.0 & -5.4725886874 & -1.2381061438 & 0.0000000000 \\
\hline $\mathrm{C}$ & 6.0 & 5.4725886874 & 1.2381061438 & 0.0000000000 \\
\hline $\mathrm{C}$ & 6.0 & -5.4725886874 & 1.2381061438 & 0.0000000000 \\
\hline $\mathrm{C}$ & 6.0 & 4.0214784718 & -1.2414960540 & 0.0000000000 \\
\hline $\mathrm{C}$ & 6.0 & -4.0214784718 & -1.2414960540 & 0.0000000000 \\
\hline $\mathrm{C}$ & 6.0 & 4.0214784718 & 1. 2414960540 & 0.0000000000 \\
\hline $\mathrm{C}$ & 6.0 & -4.0214784718 & 1.2414960540 & 0.0000000000 \\
\hline $\mathrm{C}$ & 6.0 & 6.1528546455 & -2.4750915850 & 0.0000000000 \\
\hline $\mathrm{C}$ & 6.0 & -6.1528546455 & -2.4750915850 & 0.0000000000 \\
\hline $\mathrm{C}$ & 6.0 & 6.1528546455 & 2.4750915850 & 0.0000000000 \\
\hline $\mathrm{C}$ & 6.0 & -6.1528546455 & 2.4750915850 & 0.0000000000 \\
\hline $\mathrm{C}$ & 6.0 & 3.3482476722 & -2.4788662615 & 0.0000000000 \\
\hline $\mathrm{C}$ & 6.0 & -3.3482476722 & -2.4788662615 & 0.0000000000 \\
\hline C & 6.0 & 3.3482476722 & 2.4788662615 & 0.0000000000 \\
\hline $\mathrm{C}$ & 6.0 & -3.3482476722 & 2.4788662615 & 0.0000000000 \\
\hline $\mathrm{C}$ & 6.0 & 5.4778763675 & -3.6918197777 & 0.0000000000 \\
\hline $\mathrm{C}$ & 6.0 & -5.4778763675 & -3.6918197777 & 0.0000000000 \\
\hline $\mathrm{C}$ & 6.0 & 5.4778763675 & 3.6918197777 & 0.0000000000 \\
\hline $\mathrm{C}$ & 6.0 & -5.4778763675 & 3.6918197777 & 0.0000000000 \\
\hline $\mathrm{C}$ & 6.0 & 4.0282544584 & -3.6942743126 & 0.0000000000 \\
\hline $\mathrm{C}$ & 6.0 & -4.0282544584 & -3.6942743126 & 0.0000000000 \\
\hline $\mathrm{C}$ & 6.0 & 4.0282544584 & 3.6942743126 & 0.0000000000 \\
\hline $\mathrm{C}$ & 6.0 & -4.0282544584 & 3.6942743126 & 0.0000000000 \\
\hline $\mathrm{C}$ & 6.0 & 5.4735166467 & -6.1287491908 & 0.0000000000 \\
\hline $\mathrm{C}$ & 6.0 & -5.4735166467 & -6.1287491908 & 0.0000000000 \\
\hline $\mathrm{C}$ & 6.0 & 5.4735166467 & 6.1287491908 & 0.0000000000 \\
\hline $\mathrm{C}$ & 6.0 & -5.4735166467 & 6.1287491908 & 0.0000000000 \\
\hline
\end{tabular}




\begin{tabular}{|c|c|c|c|c|}
\hline $\mathrm{C}$ & 6.0 & 4.0414062574 & -6.1309006058 & 0.0000000000 \\
\hline $\mathrm{C}$ & 6.0 & -4.0414062574 & -6.1309006058 & 0.0000000000 \\
\hline $\mathrm{C}$ & 6.0 & 4.0414062574 & 6.1309006058 & 0.0000000000 \\
\hline $\mathrm{C}$ & 6.0 & -4.0414062574 & 6.1309006058 & 0.0000000000 \\
\hline C & 6.0 & 6.1666750376 & -4.9500376145 & 0.0000000000 \\
\hline $\mathrm{C}$ & 6.0 & -6.1666750376 & -4.9500376145 & 0.0000000000 \\
\hline $\mathrm{C}$ & 6.0 & 6.1666750376 & 4.9500376145 & 0.0000000000 \\
\hline $\mathrm{C}$ & 6.0 & -6.1666750376 & 4.9500376145 & 0.0000000000 \\
\hline $\mathrm{C}$ & 6.0 & 3.3442700178 & -4.9541008366 & 0.0000000000 \\
\hline $\mathrm{C}$ & 6.0 & -3.3442700178 & -4.9541008366 & 0.0000000000 \\
\hline $\mathrm{C}$ & 6.0 & 3.3442700178 & 4.9541008366 & 0.0000000000 \\
\hline $\mathrm{C}$ & 6.0 & -3.3442700178 & 4.9541008366 & 0.0000000000 \\
\hline $\mathrm{H}$ & 1.0 & 9.8791853511 & 0.0000000000 & 0.0000000000 \\
\hline $\mathrm{H}$ & 1.0 & -9.8791853511 & 0.0000000000 & 0.0000000000 \\
\hline $\mathrm{H}$ & 1.0 & 7.2386447019 & -2.4700717770 & 0.0000000000 \\
\hline $\mathrm{H}$ & 1.0 & -7.2386447019 & -2.4700717770 & 0.0000000000 \\
\hline $\mathrm{H}$ & 1.0 & 7.2386447019 & 2.4700717770 & 0.0000000000 \\
\hline $\mathrm{H}$ & 1.0 & -7.2386447019 & 2.4700717770 & 0.0000000000 \\
\hline $\mathrm{H}$ & 1.0 & 2.2621663549 & -2.4804311889 & 0.0000000000 \\
\hline $\mathrm{H}$ & 1.0 & -2.2621663549 & -2.4804311889 & 0.0000000000 \\
\hline $\mathrm{H}$ & 1.0 & 2.2621663549 & 2.4804311889 & 0.0000000000 \\
\hline $\mathrm{H}$ & 1.0 & -2.2621663549 & 2.4804311889 & 0.0000000000 \\
\hline $\mathrm{H}$ & 1.0 & 7.2542661597 & -4.9448454951 & 0.0000000000 \\
\hline $\mathrm{H}$ & 1.0 & -7.2542661597 & -4.9448454951 & 0.0000000000 \\
\hline $\mathrm{H}$ & 1.0 & 7.2542661597 & 4.9448454951 & 0.0000000000 \\
\hline $\mathrm{H}$ & 1.0 & -7.2542661597 & 4.9448454951 & 0.0000000000 \\
\hline $\mathrm{H}$ & 1.0 & 2.2566699811 & -4.9524860584 & 0.0000000000 \\
\hline $\mathrm{H}$ & 1.0 & -2.2566699811 & -4.9524860584 & 0.0000000000 \\
\hline $\mathrm{H}$ & 1.0 & 2.2566699811 & 4.9524860584 & 0.0000000000 \\
\hline $\mathrm{H}$ & 1.0 & -2.2566699811 & 4.9524860584 & 0.0000000000 \\
\hline
\end{tabular}




$\begin{array}{lrrrr}\mathrm{H} & 1.0 & 3.5118557631 & 7.0800143782 & 0.0000000000 \\ \mathrm{H} & 1.0 & -3.5118557631 & 7.0800143782 & 0.0000000000 \\ \mathrm{H} & 1.0 & 3.5118557631 & -7.0800143782 & 0.0000000000 \\ \mathrm{H} & 1.0 & -3.5118557631 & -7.0800143782 & 0.0000000000 \\ \mathrm{H} & 1.0 & 6.0058072668 & 7.0763213807 & 0.0000000000 \\ \mathrm{H} & 1.0 & -6.0058072668 & 7.0763213807 & 0.0000000000 \\ \mathrm{H} & 1.0 & 6.0058072668 & -7.0763213807 & 0.0000000000 \\ \mathrm{H} & 1.0 & -6.0058072668 & -7.0763213807 & 0.0000000000\end{array}$




\section{NMR DATA}

${ }^{1} \mathrm{H}-\mathrm{NMR}\left(500 \mathrm{MHz}, \mathrm{CDCl}_{3}, \delta \mathrm{ppm}\right): 9.72(\mathrm{~s}, 1 \mathrm{H}), 9.41-9.33(\mathrm{~m}, 3 \mathrm{H}), 8.04-7.96$ (m, 3H), 7.77-7.75 (m, 1H), 7.48-7.45 (2H), 7.29-7.25 (m, 1H) and 1.48-1.38 (m, 42H).

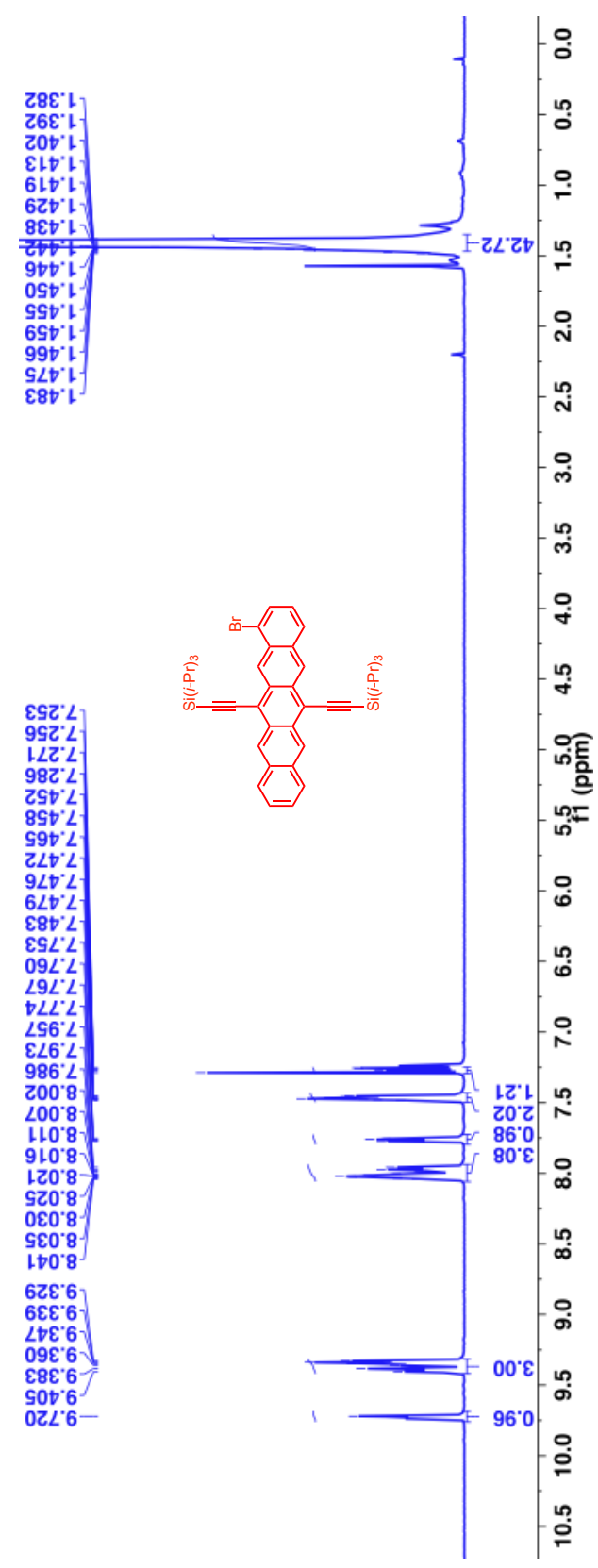


${ }^{13} \mathrm{C}-\mathrm{NMR}\left(125 \mathrm{MHz}, \mathrm{CDCl}_{3}, \delta \mathrm{ppm}\right): 132.8,132.5,132.4,130.94,130.9,130.8,132.75$, 130.72 , 129.8, 128.9, 128.7, 128.66, 127.3, 126.9, 126.6, 126.3, 126.3, 126.2, 125.8, 123.4, 119.3, 118.3, 108.0, 107.5, 104.5, 104.3, 19.1, 19.0 and 11.7.

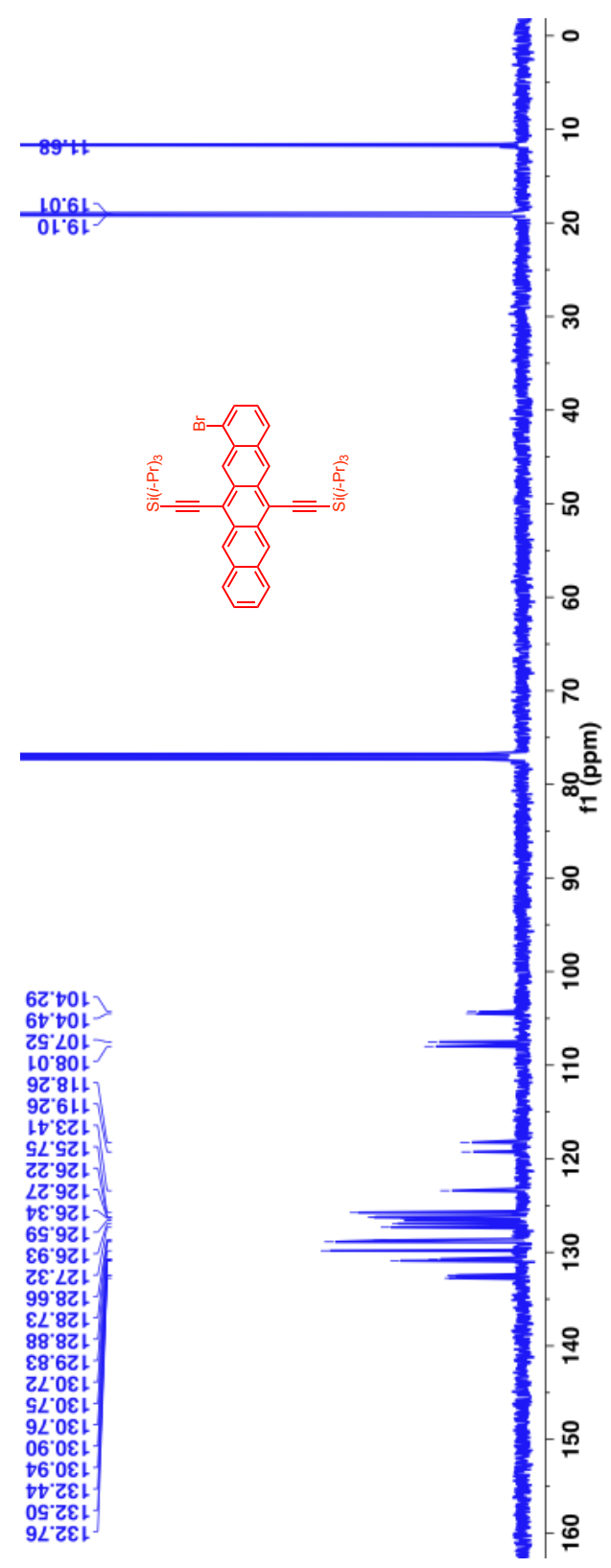


${ }^{1} \mathrm{H}-\mathrm{NMR}\left(400 \mathrm{MHz}, \mathrm{CDCl}_{3}, \delta \mathrm{ppm}\right): 9.93$ (s, 1H), 9.52 (s, 1H), 9.37 (s, 2H), 8.12-8.08 (m, 2H), 8.04-8.00 (m, 2H), 7.47-7.44 (m, 3H), $1.54(\mathrm{~s}, 12 \mathrm{H})$ and 1.44-1.40 (m, 42H).

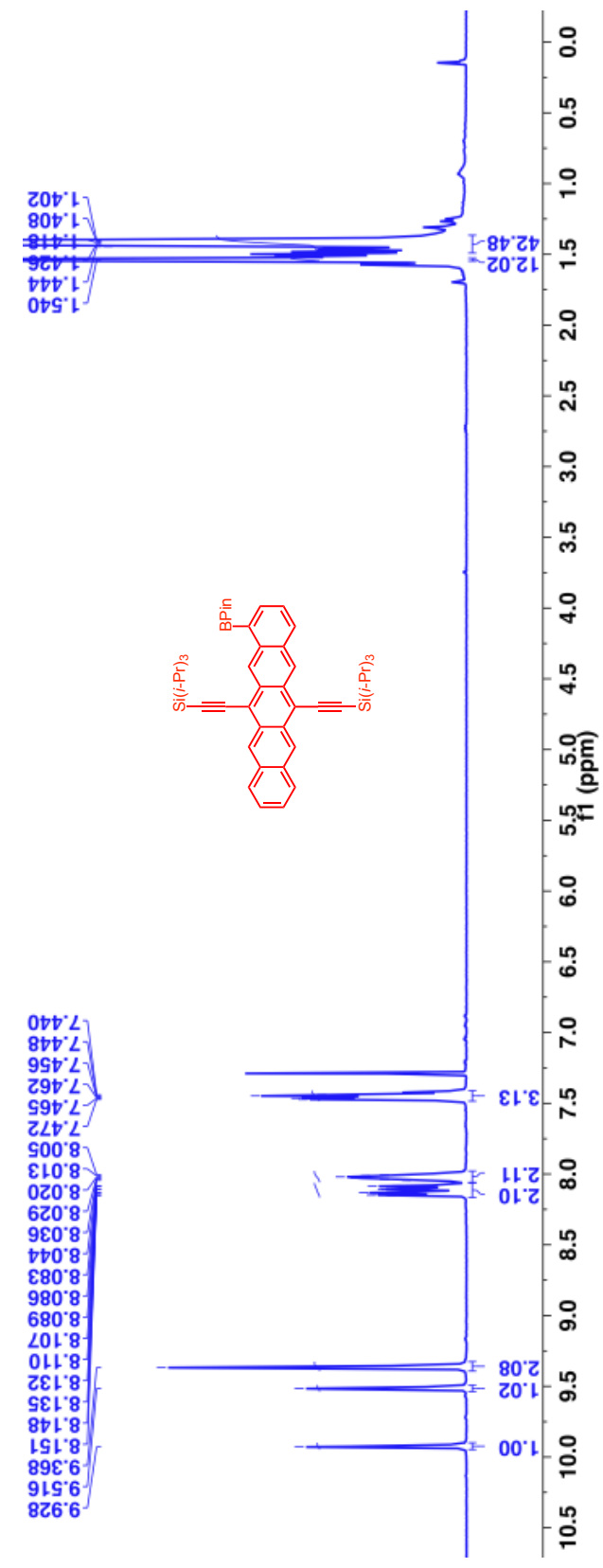


${ }^{13} \mathrm{C}-\mathrm{NMR}\left(100 \mathrm{MHz}, \mathrm{CDCl}_{3}, \delta \mathrm{ppm}\right):$ 137.4, 134.5, 132.6, 132.4, 132.3, 132.2, 131.6, 130.7, 130.6, 130.4, 128.6, 127.3, 126.8, 126.7, 126.2, 125.96, 125.1, 118.9, 118.3, 107.6, 107.0, 105.5, $104.9,83.9,24.9,19.2,19.0,12.0$ and 11.7.

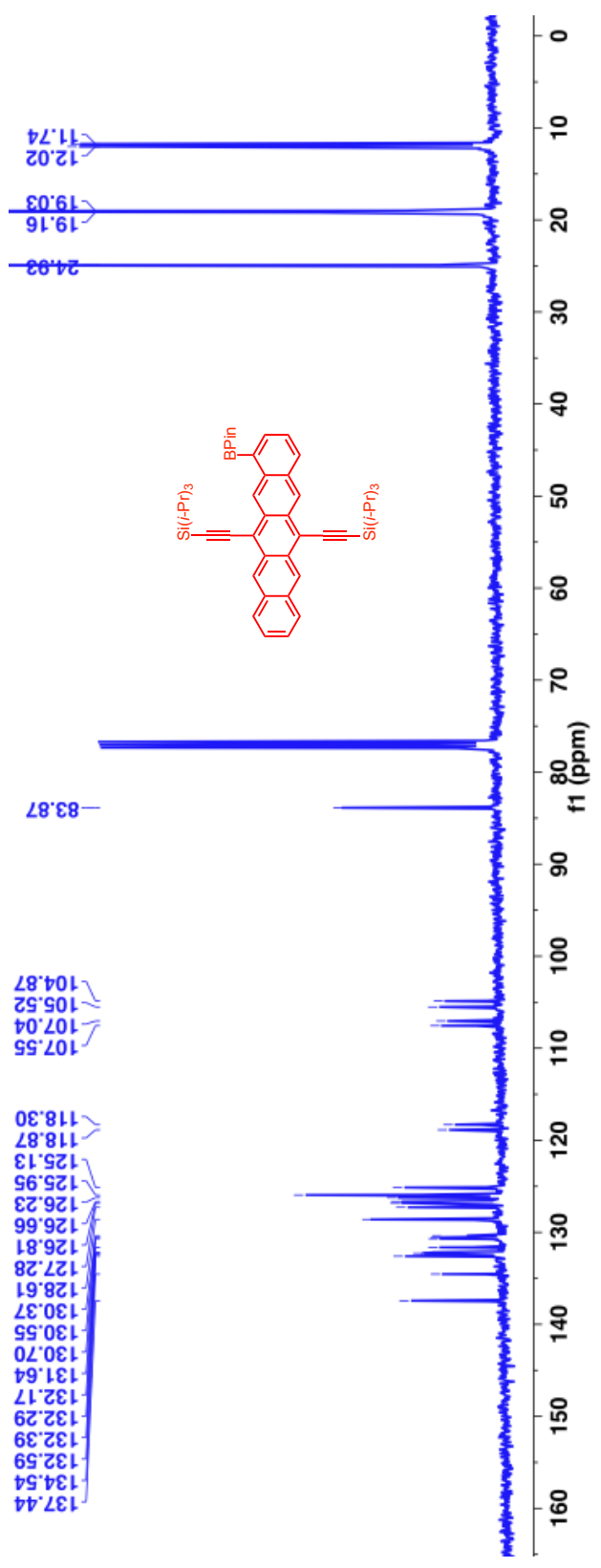


${ }^{1} \mathrm{H}-\mathrm{NMR}\left(500 \mathrm{MHz}, \mathrm{CDCl}_{3}, \delta \mathrm{ppm}\right): 9.51(\mathrm{~s}, 1 \mathrm{H}), 9.31(\mathrm{~s}, 1 \mathrm{H}), 9.15(\mathrm{~s}, 1 \mathrm{H}), 9.07(\mathrm{~s}, 1 \mathrm{H})$, $8.15(\mathrm{~d}, J=8.5 \mathrm{~Hz}, 1 \mathrm{H}), 7.97(\mathrm{~d}, J=8.5 \mathrm{~Hz}, 1 \mathrm{H}), 7.85(\mathrm{~d}, J=8.5 \mathrm{~Hz}, 1 \mathrm{H}), 7.61-7.58$ $(\mathrm{m}, 1 \mathrm{H}), 7.52-7.51(\mathrm{~m}, 1 \mathrm{H}), 7.41-7.34(\mathrm{~m}, 2 \mathrm{H}), 1.496-1.44(\mathrm{~m}, 21 \mathrm{H}), 0.87-0.86(\mathrm{~m}, 9 \mathrm{H})$ and $0.76-0.68(\mathrm{~m}, 12 \mathrm{H})$.

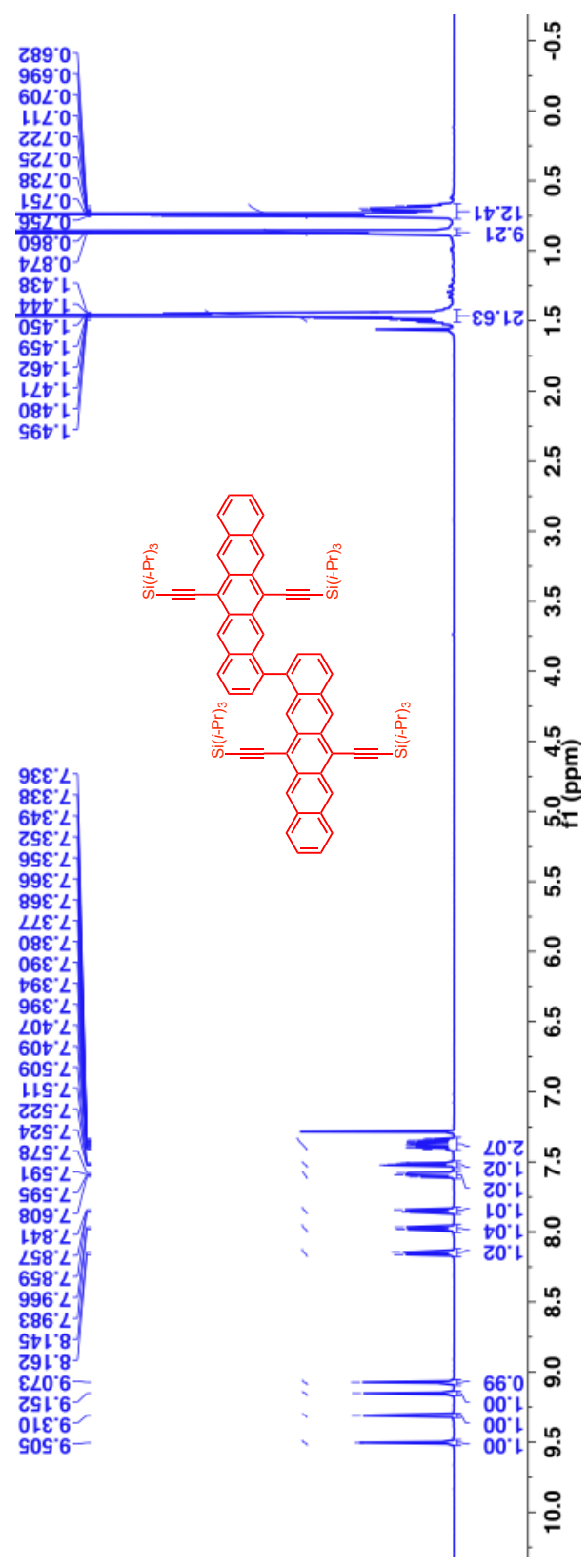


${ }^{13} \mathrm{C}-\mathrm{NMR}\left(125 \mathrm{MHz}, \mathrm{CDCl}_{3}, \delta \mathrm{ppm}\right):$ 139.3, 132.7, 132.3, 132.2, 132.1, 130.8, 130.6, 129.1, 128.7, 128.6, 128.2, 126.9, 126.5, 126.1, 125.9, 125.9, 125.8, 125.7, 118.9, 117.9, 107.4, $106.8,104.9,103.8,19.1,18.6,18.5,11.8$ and 11.2.

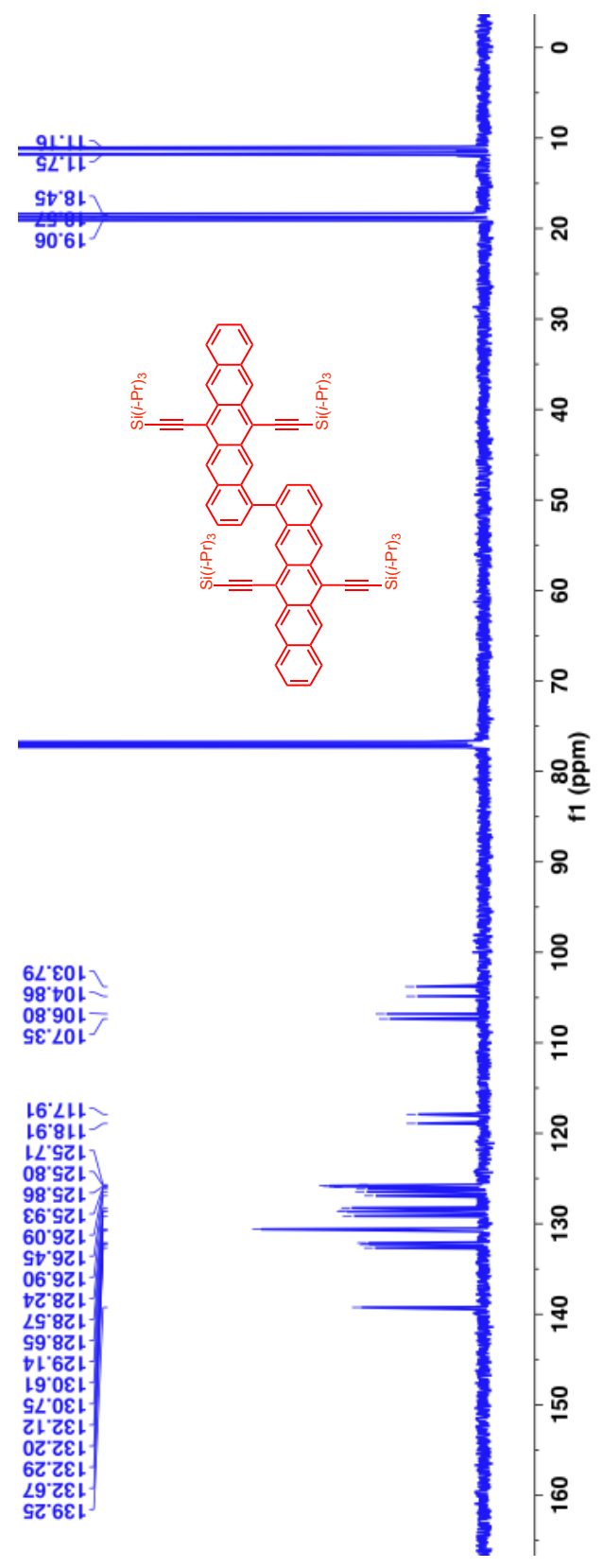


${ }^{1} \mathrm{H}-\mathrm{NMR}\left(500 \mathrm{MHz}, \mathrm{CDCl}_{3}, \delta \mathrm{ppm}\right)$ : 9.32-9.23 (m, 8H), 8.02-7.97 (m, 8H), 7.86-7.84 $(\mathrm{m}, 2 \mathrm{H}), 7.50(\mathrm{~s}, 2 \mathrm{H}), 7.49-7.42(\mathrm{~m}, 4 \mathrm{H}), 1.85-1.77(\mathrm{~m}, 8 \mathrm{H}), 1.58-1.54\left(8 \mathrm{H}\right.$, overlap $\left.\mathrm{H}_{2} \mathrm{O}\right)$, 1.42-1.34 (m, 70H), 1.29-1.27 (m, 16H), 1.03-0.98 (m, 9H) and 0.89-0.81 (m, 13H)

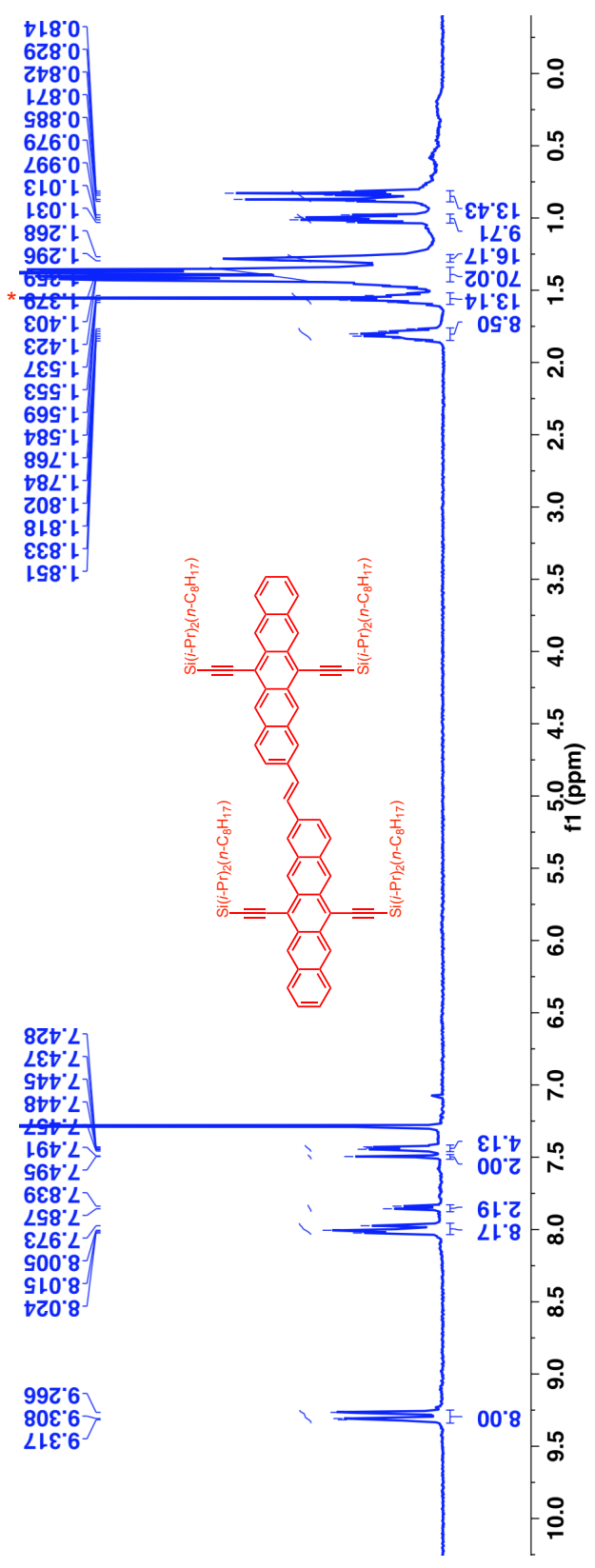


${ }^{13} \mathrm{C}-\mathrm{NMR}\left(125 \mathrm{MHz}, \mathrm{CDCl}_{3}, \delta \mathrm{ppm}\right): 134.6,132.4,132.3,132.2,131.9,131.0,130.8$, 130.7, 130.5, 129.1, 128.6, 128.2, 126.4, 126.3, 126.2, 126.0, 125.99, 125.91, 123.1, 118.4, 118.2, 107.4, 107.3, 104.6, 104.5, 34.1, 34.07, 32.0, 29.6, 29.5, 29.44, 29.41, 25.0, 24.99, 22.7, $18.8,18.77,18.72,18.5,18.49,14.1,14.08,12.3,12.2,10.6$ and 10.5 .

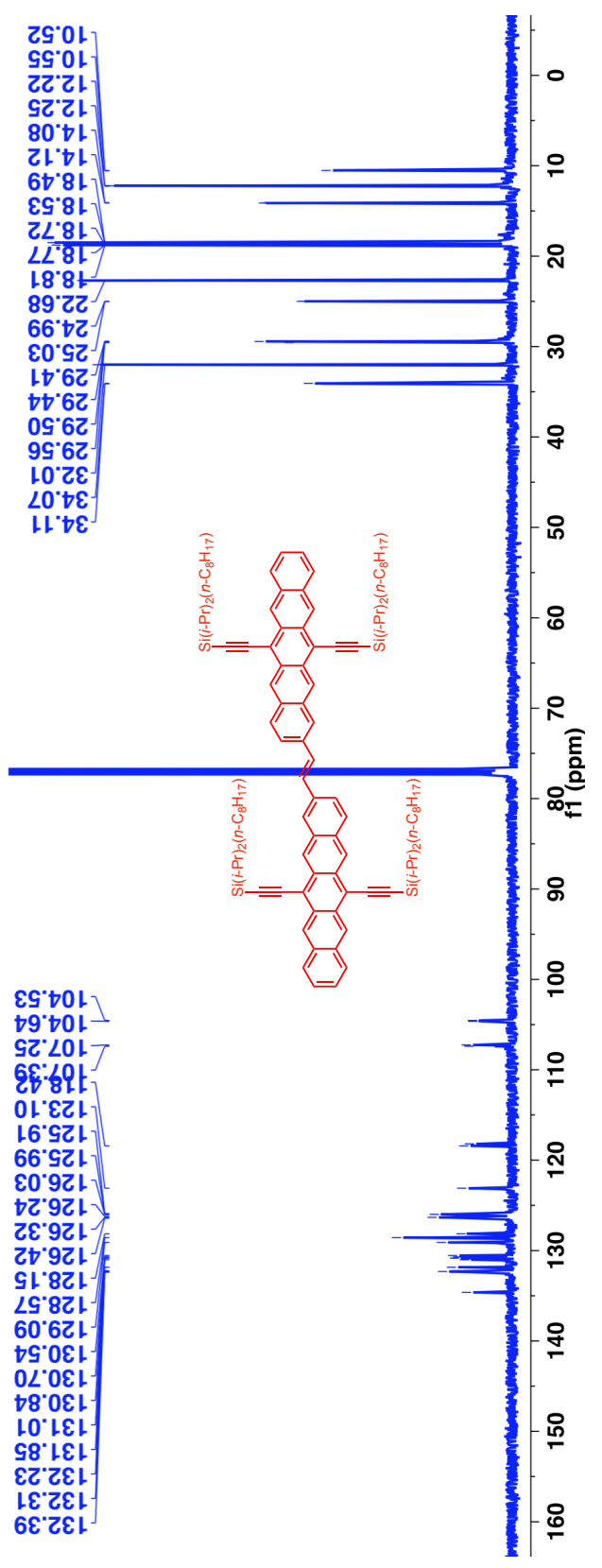


[1] Fuemmeler, E. G., Sanders, S. N., Pun, A. B., Kumarasamy, E., Zeng, T., Miyata, K., Steigerwald, M. L., Zhu, X.-Y., Sfeir, M. Y., Campos, L. M., and Ananth, N. (2016) A Direct Mechanism of Ultrafast Intramolecular Singlet Fission in Pentacene Dimers. ACS Cent. Sci. 2, 316-324.

[2] Zeng, T., Hoffmann, R., and Ananth, N. (2014) The Low-Lying Electronic States of Pentacene and Their Roles in Singlet Fission. J. Am. Chem. Soc. 136, 5755-5764.

[3] Granovsky, A. A. (2011) Extended multi-configuration quasi-degenerate perturbation theory: The new approach to multi-state multi-reference perturbation theory. J. Chem. Phys. 134, 214113.

[4] Aryanpour, K., Psiachos, D., and Mazumdar, S. (2010) Theory of interfacial charge-transfer complex photophysics in $\pi$-conjugated polymer-fullerene blends. Phys. Rev. B 81, 085407.

[5] Venkataraman, L., Klare, J. E., Nuckolls, C., Hybertsen, M. S., and Steigerwald, M. L. (2006) Dependence of single-molecule junction conductance on molecular conformation. Nature 442, 904-907.

[6] Mataga, N., and Nishimoto, K. (1957) Electronic Structure and Spectra of Nitrogen Heterocycles. Z. Phys. Chem. 13, 140-157.

[7] Zollinger, H. Color chemistry: syntheses, properties, and applications of organic dyes and pigments; John Wiley \& Sons, 2003.

[8] Geskin, V. M., Lambert, C., and Brédas, J.-L. (2003) Origin of High Second- and ThirdOrder Nonlinear Optical Response in Ammonio/Borato Diphenylpolyene Zwitterions: the Remarkable Role of Polarized Aromatic Groups. J. Am. Chem. Soc. 125, 15651-15658.

[9] Schmidt, M. W., Baldridge, K. K., Boatz, J. A., Elbert, S. T., Gordon, M. S., Jensen, J. H., Koseki, S., Matsunaga, N., Nguyen, K. A., Su, S., Windus, T. L., Dupuis, M., and Montgomery, J. A. (1993) General atomic and molecular electronic structure system. J. Comput. Chem. 14, 1347-1363.

[10] Gordon, M. S., and Schmidt, M. W. In Theory and Applications of Computational Chemistry; Dykstra, C. E., Frenking, G., Kim, K. S., and Scuseria, G. E., Eds.; Elsevier: Amsterdam, 
2005; pp $1167-1189$.

[11] Towns, J., Cockerill, T., Dahan, M., Foster, I., Gaither, K., Grimshaw, A., Hazlewood, V., Lathrop, S., Lifka, D., Peterson, G. D., Roskies, R., Scott, J. R., and Wilkens-Diehr, N. (2014) XSEDE: Accelerating Scientific Discovery. Comput. Sci. Eng. 16, 62-74.

[12] Pariser, R. (1956) Theory of the Electronic Spectra and Structure of the Polyacenes and of Alternant Hydrocarbons. J. Chem. Phys. 24, 250-268.

[13] Coulson, C. A., and Rushbrooke, G. S. (1940) Note on the method of molecular orbitals. Math. Proc. Cambridge 36, 193-200.

[14] Lehnherr, D., Murray, A., McDonald, R., and Tykwinski, R. (2010) A Modular Synthetic Approach to Conjugated Pentacene Di-, Tri-, and Tetramers. Angew. Chem., Int. Ed. 49, 6190-6194.

[15] Kumarasamy, E., Sanders, S. N., Pun, A. B., Vaselabadi, S. A., Low, J. Z., Sfeir, M. Y., Steigerwald, M. L., Stein, G. E., and Campos, L. M. (2016) Properties of Poly- and Oligopentacenes Synthesized from Modular Building Blocks. Macromolecules 49, 1279-1285.

[16] Sanders, S. N., Kumarasamy, E., Pun, A. B., Trinh, M. T., Choi, B., Xia, J., Taffet, E. J., Low, J. Z., Miller, J. R., Roy, X., Zhu, X.-Y., Steigerwald, M. L., Sfeir, M. Y., and Campos, L. M. (2015) Quantitative Intramolecular Singlet Fission in Bipentacenes. J. Am. Chem. Soc. 137, 8965-72. 